\title{
Norois
}

Environnement, aménagement, société

\section{De Villeneuve-d'Ascq Technopole à la métropole technopolitaine : espace, réseaux, gouvernance}

From "Villeneuve-d'Ascq Technology parks" to a technological metropolitan area: space, networks, governance

Christine Liefooghe, Sylvie Delmer, Maryvonne Prevot, Isabelle Estienne et Karine Stachowski

\section{CpenEdition}

\section{Journals}

Édition électronique

URL : http://journals.openedition.org/norois/1806

DOI : $10.4000 /$ norois. 1806

ISBN : 978-2-7535-1548-2

ISSN : 1760-8546

\section{Éditeur}

Presses universitaires de Rennes

\section{Édition imprimée}

Date de publication : 1 septembre 2006

ISBN : 978-2-7535-0342-7

ISSN : 0029-182X

\section{Référence électronique}

Christine Liefooghe, Sylvie Delmer, Maryvonne Prevot, Isabelle Estienne et Karine Stachowski, «De Villeneuve-d'Ascq Technopole à la métropole technopolitaine : espace, réseaux, gouvernance ", Norois [En ligne], 200 | 2006/3, mis en ligne le 12 décembre 2008, consulté le 01 mai 2019. URL : http:// journals.openedition.org/norois/1806 ; DOI : 10.4000/norois.1806 


\title{
De Villeneuve-D’Asce Technopole
}

\section{À LA MÉTROPOLE TECHNOPOLITAINE : ESPACE, RÉSEAUX, GOUVERNANCE}

\author{
Christine Liefooghe, Sylvie Delmer, Maryvonne Prevot, \\ IsABelle Estienne, Karine StaCHOWSKI \\ UNIVERSITÉ DE LILLE 1 \\ TVES, EA 4019 - CNRS \\ christine.liefooghe@univ-lille1.fr
}

\section{RÉSUMÉ}

Villeneuve-d'Ascq Technopole est un projet politique local qui a rencontré, en 1986, l'adhésion des mondes de la recherche et de l'entreprise dans un contexte institutionnel acquis depuis peu au modèle du développement par l'innovation technologique. Marketing territorial et animation des réseaux d'acteurs ont pour fonction d'accroître l'attractivité de la ville et d'y faire émerger un milieu technopolitain. Ce projet territorial conforte des options d'aménagement initiées par l'opération Ville Nouvelle voire même par l'exurbanisation des universités lilloises dans les années 1960. Imaginée à l'origine comme le moteur de la reconversion régionale, Villeneuve-d'Ascq Technopole n'est plus aujourd'hui qu'un des territoires à vocation technopolitaine de la métropole lilloise. Ce changement d'échelle introduit une concurrence entre les technopôles métropolitains, ce qui rend plus nécessaire un fonctionnement en réseau des acteurs de l'innovation au service de la dynamique technopolitaine du Nord-Pas-de-Calais.

Mots CLÉs : Innovation - Nord/Pas-de-Calais - Technopole - Ville nowvelle.

\footnotetext{
ABSTRACT

From "Villeneuve-d'Ascq Technology parks" to a technological metropolitan area: space, networks, governance

"Villeneuve-d'Ascq Technopole" is a local political project founded in 1986 with the support from business and research world, in an institutional context lately dedicated to the model of development through technological innovation. Territorial marketing and actors networking aim to increase the attractivity of the city and the emergence of an innovative milieu. This project reinforces town planning options adopted during the New Town period, following the exurbanization of Lille universities in the 1960s. First conceived as the development pole for the regional economic regeneration, "Villeneuve-d'Ascq Technopole" is nowadays in competition with different science or technological parks within the Lille metropolitan area. Nevertheless, the cooperation between local actors aims to improve economic innovations in the Nord/Pas-de-Calais region.
}

KEY WORDS : Innovation - New Town - Nord/Pas-de-Calais (north of France) - Science Park. 
En 1986, Gérard Caudron, maire de Villeneuve-d’Ascq, déclare : « Nous ne sommes plus une ville nouvelle, mais nous devons rester différents, être imaginatifs, avoir un projet, faire parler de nous ${ }^{1}$. » Entrer dans le cercle restreint des technopoles qui s'édifient à la frange des grandes villes françaises au milieu des années 1980 est l'ambition d'un maire qui souhaite faire de Villeneuved'Ascq le moteur de la reconversion du Nord-Pas-de-Calais. Se vendre à l'international, attirer des entreprises de haute technologie, favoriser l'éclosion d'un milieu innovateur, autant d'objectifs susceptibles de transformer une ville nouvelle inachevée en «technopole verte » où il fait bon vivre et travailler. Qu'est-il advenu de cette ambition? Villeneuve-d'Ascq est-elle une technopole fonctionnelle, vingt ans après le lancement du projet et au-delà du marketing territorial ? Difficile de répondre à cette question, faute d'études approfondies sur la réalité d'un fonctionnement de type milieu innovateur ou cluster. L'échelle communale est-elle d'ailleurs la mieux adaptée pour évaluer les relations entre universités et entreprises, ainsi que la diffusion de l'esprit d'innovation et d'entreprenariat? Nous avons donc pris le parti, dans un premier temps, de montrer le poids des héritages en matière de décisions politiques, de choix urbanistiques et paysagers, au-delà des discours politiques sur la nouveauté du projet de technopole. L'aventure technopolitaine de Villeneuve-d'Ascq s'inscrit dans le temps long de l'aménagement et du développement de l'agglomération de Lille-Roubaix-Tourcoing, et le bilan du projet villeneuvois doit se lire à cette échelle (fig. 1). Néanmoins, la construction d'un espace technopolitain restreint au regroupement spatial, sur une seule commune, d'entreprises de haute technologie en interrelation s'oppose, à partir du milieu des années 1990, à l'ambition technopolitaine de la métropole lilloise. Une technopole multipolaire émerge, articulée autour de technopôles, parcs dédiés aux entreprises de haut niveau technologique. Ce schéma, qui s'élabore par étapes, brouille les cartes du projet politique et économique de Villeneuve-d'Ascq et invalide de fait l'analyse de la dynamique technopolitaine à cette seule échelle. L'article pose donc en second lieu la question de l'articulation entre espaces, réseaux et gouvernance dans l'inscription territoriale de projets politiques construits à partir du concept de technopole. Le développement économique ne se décrète pas; l'existence d'un milieu innovateur ou d'un cluster encore moins. Une dynamique technopolitaine se construit cependant à l'échelle de la métropole, par un travail de mise en réseau des acteurs de la recherche, de l'innovation, de l'entreprenariat et de la gestion des territoires. Des projets d'aménagement de technopôles accompagnent ce mouvement résiliaire. Le tout s'élabore dans un esprit de coopération métropolitaine, mais aussi de compétition interne à tous les échelons institutionnels et territoriaux. Cette complexité pose alors la question de la gouvernance des réseaux et des espaces technopolitains en devenir d'une métropole lilloise qui s'engage en outre dans de multiples coopérations avec les autres territoires du Nord-Pas-de-Calais.

\section{Villeneuve-d'Ascq, une ville nouvelle programmée pour la qualité et l'innovation}

Lors de la croissance économique et démographique des années 1960, des schémas d'aménagement de l'agglomération lilloise prévoient d'en faire le pôle de développement de la région et une « métropole d'équilibre ». La création d'une ville nouvelle à l'est de Lille est une des options envisagées pour maîtriser l'extension urbaine et façonner un cadre de vie de qualité, dans un espace programmé pour la recherche et l'innovation dès les prémices de la réflexion. Débats conceptuels, choix techniques et décisions politiques ont présidé pendant plus de vingt ans, non sans heurts, à la naissance de Villeneuve-d'Ascq ${ }^{2}$.

1. Archives du journal municipal de Villeneuve-d'Ascq [www.rassemblementcitoyen.org].

2. On pourra s'aider des figures 1 et 3 pour situer les lieux évoqués dans cette partie historique. 

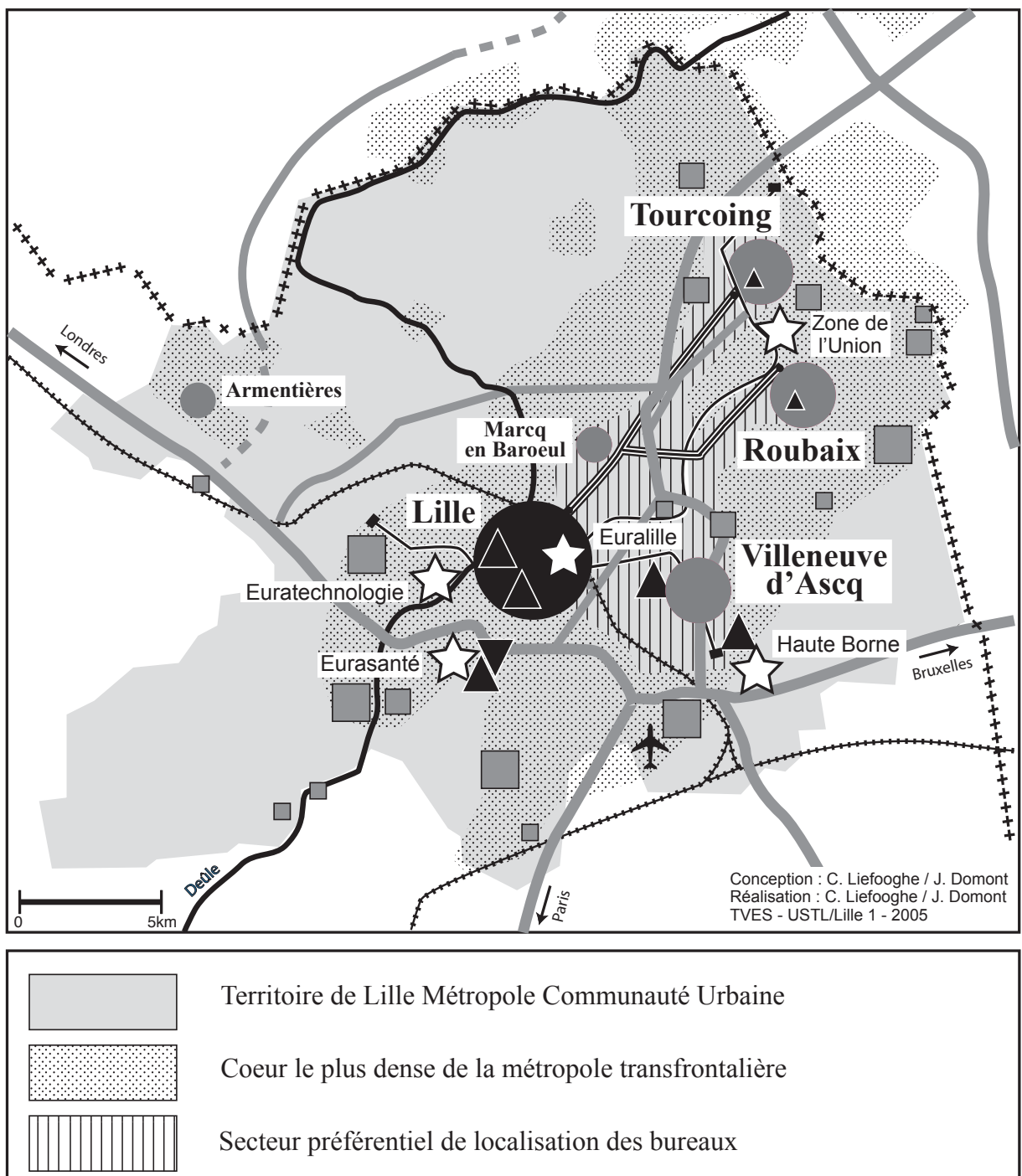

Territoire de Lille Métropole Communauté Urbaine

Coeur le plus dense de la métropole transfrontalière

Secteur préférentiel de localisation des bureaux

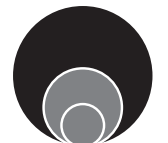

Principal pôle urbain tertiaire

Autres grands pôles urbains

Pôle d'excellence métropolitain

$\triangle \quad$ Pôles universitaires

C.H.R.U.

Principales zones d'activités

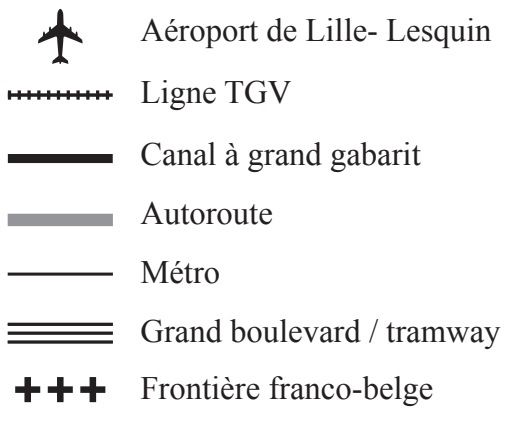

Figure 1: «Pôles d'excellence » et pôles de recherche de la métropole lilloise

Growth Poles and public Research Centers in the Lille metropolitan Area 


\section{L'EXURBANISATION DE L'UNIVERSITE : UN ACTE FONDATEUR}

L'exurbanisation de l'université résulte du débat ouvert dans les années 1950 sur la nécessité d'agrandir la faculté des Sciences de Lille pour répondre à la hausse des effectifs étudiants et au manque consécutif de locaux et de matériels (Parreau, 1996). Les partisans du maintien de l'université en ville plaident pour la rénovation de sites intra-urbains. Le recteur G. Debeyre défend au contraire l'idée de l'exurbanisation d'une « grande Cité scientifique destinée à l'enseignement et à la recherche afin d'assurer le développement du Nord par une formation professionnelle et scientifique de très haut niveau » (Baudelle, 1984). L'ouverture de l'université aux milieux économiques est l'autre pendant de la vision de G. Debeyre, par ailleurs président du Comité régional d'expansion économique. Soutenu au sommet de l'État, le recteur tranche le débat en faveur de son projet de campus sur le site d'Annappes, espace rural de l'est lillois où est prévue, à terme, la réalisation d'un nœud autoroutier. Validée par le ministère de l'Éducation Nationale et inscrite au plan d'équipement, la construction de la Cité scientifique s'engage difficilement, en butte à l'hostilité des agriculteurs expropriés. La première rentrée universitaire a lieu en 1964, dans des conditions déplorables; la première tranche de construction n'est achevée qu'en 1967. En dépit de ces aléas, le recteur décide, dès 1964, le transfert des facultés de Lettres et de Droit sur la commune de Flers, à proximité d'Annappes.

Le débat sur l'intégration de l'université dans la ville commence alors. La Cité scientifique en construction est reliée à Lille par une simple route et les transports en commun sont très insuffisants. Des bâtiments éparpillés, de grandes surfaces engazonnées et sans aucun arbre : cette copie maladroite des campus anglo-saxons est critiquée par les adversaires du projet. À la même époque, un débat national remet en cause le modèle de campus adopté pour répondre à la démocratisation de l'enseignement supérieur, et prône l'intégration des étudiants à la vie urbaine. Mais le recteur G. Debeyre revendique une démarche prospective et déclare en 1967 : « Nous avons travaillé pour l'an 2000. Le désert environnant sera entouré d'écoles d'ingénieurs, d'IUT de recherche appliquée, que l'industrie peut installer à proximité des laboratoires de recherche fondamentale » (Baudelle, 1984). En attendant, il s'agit de rompre à court terme l'isolement de la Cité scientifique, de maîtriser l'urbanisation anarchique de l'est lillois et de donner une ville à l'université.

\section{UNE VILLE NOUVELLE POUR LA RECHERCHE ET L’INNOVATION}

L'alternative entre gestion de la croissance urbaine et gestion de l'héritage industriel constitue la trame des débats contradictoires qui ont présidé dès les années 1960 aux choix d'aménagement de la conurbation de Lille-Roubaix-Tourcoing. Les plans envisagés visent à résorber l'habitat insalubre des pôles historiques et à contrer le développement radioconcentrique spontané de l'agglomération en proposant une extension urbaine vers le sud, en direction du bassin minier (Delbar, 1977). L'est lillois, longtemps protégé de l'urbanisation par des problèmes d'écoulement des eaux, n'est dans ces plans qu'un élément mineur de la recomposition spatiale de l'agglomération. Pourtant, la crise du logement et le faible prix du foncier de cet espace rural y favorisent, à partir de 1960, la réalisation non coordonnée de projets immobiliers d'envergure : grands ensembles de HLM du quartier Babylone à Flers et du quartier Résidence à Annappes; opération privée du « Domaine de Brigode ", autour d'un golf, pour attirer des cadres supérieurs dans la région. La construction de la Cité scientifique près du village d'Annappes dans les années 1960 engendre une controverse sur l'aménagement concerté de l'est lillois. L'ampleur des investissements nécessaires et l'urgence des problèmes à régler incitent l'État à imposer dès 1966 la création d'une ville nouvelle. Cette opération d'urbanisme d'envergure, sans précédent dans la région, permet de disposer de financements exceptionnels et des moyens techniques nécessaires à l'élaboration d'un projet novateur et cohérent. Elle bouleverse néanmoins les schémas locaux d'aménagement.

Dès 1966 en effet, l'OREAM-Nord (1969) a pour mission de renouveler la réflexion sur l'avenir de la capitale régionale, dans le cadre de la politique des métropoles d'équilibre. Transformer l'ag- 
glomération tripolaire en une véritable métropole d'un à deux millions d'habitants, qui devienne le ressort du renouvellement économique de toute la région, est le défi proposé par cette jeune équipe pluridisciplinaire. Cette détermination inspire un parti d'aménagement centré sur la création d'une « aire urbaine centrale ». Face à l'ampleur de la crise économique pressentie, l'idée est de réunir en une entité unique l'ancien bassin minier et la métropole lilloise pour mieux dynamiser les territoires en difficulté. Ce pari repose sur la mise en œuvre d'une politique d'amélioration du cadre de vie, en direction des paysages et de l'habitat, ainsi que d'une politique de développement des formations et d'incitation à l'innovation. Si le schéma prospectif de l'OREAM-Nord s'inspire en partie des réflexions engagées au préalable sur l'aménagement de l'agglomération lilloise, il est aussi contraint d'intégrer la Ville Nouvelle de Lille-Est envisagée par l'État, et lui accorde un rôle stratégique en matière d'enseignement supérieur, de recherche et d'industries de pointe. L'ampleur du projet de ville nouvelle va par la suite mobiliser les énergies et faire passer au second plan la réalisation de « l'aire urbaine centrale ». Inventer un nouvel urbanisme, oser de nouvelles formes architecturales, attirer des populations nouvelles et des activités innovantes sont au cœur du projet de la Ville Nouvelle de Lille-Est. L'intégration de la Cité scientifique au tissu urbain en construction est une question récurrente de l'aménagement de Villeneuve-d'Ascq ${ }^{3}$.

\section{URBANITÉ VERSUS ESPACE DÉDIÉ À LA RECHERCHE}

Comment rompre l'isolement de la Cité scientifique? Le choix est binaire : faire s’interpénétrer la ville et l'université en périphérie du campus, ou urbaniser le campus en y introduisant services divers, équipements publics et logements sur le modèle de la ville universitaire de Louvain-laNeuve en Belgique. L'université des Sciences et Techniques (USTL) s'oppose à plusieurs reprises à l'introduction d'une mixité fonctionnelle sur le campus, par crainte de voir se réduire l'espace disponible pour son propre développement. Quant au no man's land entre la Cité scientifique et le bourg d'Annappes, il est comblé par la construction du quartier du Triolo et de son centre commercial. La ville se contente donc de jouxter la Cité scientifique, des plantations séparant même les deux entités. Ce campus universitaire offre aujourd'hui d'amples espaces verts sur lesquels sont juxtaposés immeubles d'enseignement, résidences et restaurants universitaires, organisés selon une trame orthogonale autour de la bibliothèque centrale. La création d'un important réseau de haies et de brise-vent entreprise par l'Université à la fin des années 1970 permet aux usagers de bénéficier d'un cadre de vie agréable et de grande qualité environnementale. Cette générosité initiale s'est quelque peu perdue au fil des opérations de constructions qui se sont succédé depuis les années 1980. Si la Cité scientifique est désormais rattrapée de tous côtés par l'urbanisation, la ville, en tant que mixité d'activités, n'est pas encore parvenue à s'y installer : le campus reste dans sa globalité un ensemble monofonctionnel.

La rétrocession à la ville, au milieu des années 1980, de terrains appartenant à l'USTL pour réaliser le Parc d'innovation de la Haute-Borne marque un tournant dans les relations entre les aménageurs et les universitaires. Mais la question du lien entre ville et site dédié à la recherche ressurgit à travers la réalisation en cours du parc scientifique européen de la Haute-Borne (à ne pas confondre avec le précédent). Cet espace (fig. 2) a pour projet l'intégration harmonieuse de laboratoires, de parcs d'entreprises, de zones d'habitat et de services dans un cadre respectueux de l'environnement, en préservant notamment des parcelles d'exploitation agricole. Les concepteurs vantent l'urbanité offerte par la ville, ses commerces, ses loisirs, ses logements. Pour le moment, la réalisation est loin de ces ambitions initiales, en raison du manque de desserte par transports en commun et des coupures physiques très nettes entre le parc scientifique de la Haute-Borne et la Cité scientifique. De plus, la zone d'habitat censée créer du lien entre le parc et le tissu urbain ancien lui tourne le dos. Quant aux services, ils se limitent à un hôtel d'accueil pour les chercheurs. La problématique de l’intégration de

3. La Ville Nouvelle de Lille-Est devient Villeneuve-d'Ascq en 1970 suite à la fusion des communes d'Ascq, Annappes et Flers. 
la Cité scientifique à la ville se reproduit donc presque à l'identique, trente ans après le lancement de l'opération ville nouvelle censée résoudre la question.

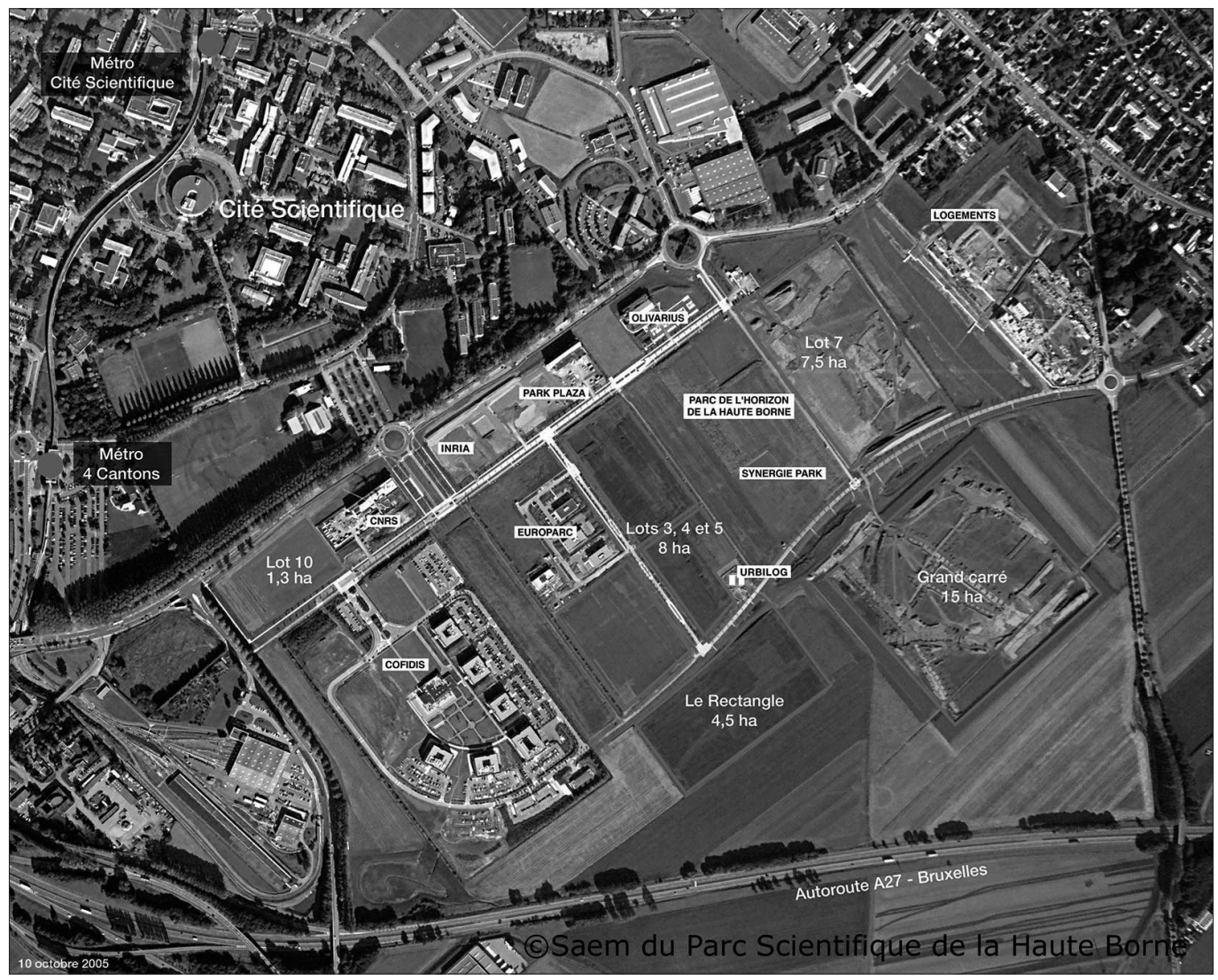

Figure 2 : «Villeneuve-d'Ascq Technopole verte » : la Cité scientifique et le Parc scientifique européen de la HauteBorne (crédit photo SAEM Haute-Borne)

Science Park

Villeneuve-d'Ascq, The "Green Technopole": the scientific University and the Haute-Borne european

\section{LA QUALITÉ DU CADRE DE VIE AU SERVICE DE L’INNOVATION TECHNOLOGIQUE}

Les objectifs assignés à la ville nouvelle de Lille-Est étaient ambitieux : accueillir 100000 habitants et 40000 étudiants à l'horizon 1985, y installer des équipements à rayonnement métropolitain et régional, créer un cadre urbain vivant et attractif, et devenir un pôle de recherche et d'innovation pour la région. Le paysage est au cœur d'un projet imaginé en opposition à l'urbanisme des ZUP, celui d'une « ville à la campagne » qui offre un nouvel art de vivre. Un site ingrat de marais et de zones inondables a servi de prétexte à une succession de lacs (Barles, 2005), de parcs et de collines artificielles se lovant dans un espace de moins en moins urbanisé, jusqu'à rejoindre vers l'est la vallée de la Marque et ses terres agricoles. Un patrimoine systématiquement préservé, un maillage piétonnier dense et inscrit dans un écrin de verdure contribuent à la qualité environnementale, à l'origine de l'image de «ville verte». Mais la ville nouvelle se doit aussi d'offrir des emplois pour éviter les migrations pendulaires domicile/travail. Le premier schéma de secteur de Lille-Est, en 1967, prévoit des zones d'activités en périphérie, à l'imitation des premières zones industrielles 
locales (Seclin, Lesquin, la Pilaterie). Les urbanistes des années 1960 considèrent en effet les usines comme le «cancer de la cité », et une vision fonctionnaliste et hygiéniste s'impose dans une agglomération lilloise historiquement constituée par une imbrication complexe de l'industrie et de l'habitat (Anonyme, 1969). Dans la même ligne de pensée, les zones d'activités intégrées au tissu urbain de la ville nouvelle, lors de la révision du schéma en 1970, sont dédiées aux bureaux et aux industries de pointe non polluantes. Cherchant avant tout à vendre sa «ville verte », l'Établissement Public d'Aménagement de Lille-Est (EPALE) applique aux zones d'activités banales, ainsi qu'à toutes les franges urbaines, une politique de masque arboré (Estienne, 2004). Il leur impose, de plus, des quotas de plantations, également valables pour les espaces de stationnement. Mais la crise du milieu des années 1970 entraine la mévente des grandes zones industrielles. L'EPALE (1978) y répond par la dissémination des activités économiques dans le tissu urbain, en s'adaptant à la demande des artisans, PME et sociétés de service. La qualité architecturale des établissements devient progressivement un facteur essentiel d'intégration dans le bâti résidentiel. Dans ce processus évolutif s'élabore un modèle de parc d'entreprises, dont l'aboutissement actuel est la construction du Parc Scientifique de la Haute-Borne selon les normes «Haute Qualité Environnementale».

Pour faire de la ville nouvelle de Lille-Est le pôle d'innovation du Nord-Pas-de-Calais, deux zones sont réservées aux activités de pointe : elles accueillent deux centres de recherche, l'ITF (Institut Textile de France) et le CERTIA (agro-alimentaire). On imagine pouvoir reproduire le modèle de la « route 128 » de Boston, ville d'industrie textile en crise reconvertie dans l'électronique. À Villeneuve-d'Ascq (fig. 3), les laboratoires et entreprises de haute technologie sont censés s'égrener le long de l'autoroute intra-urbaine reliant la Cité scientifique et sa réserve foncière de la HauteBorne, au sud, et les zones du Tir à Loques et du Recueil, au nord. Au début des années 1970, l'État donne l'impulsion nécessaire à la création ou à la délocalisation de centres de recherche sur Lille-Est. Des ministres s'intéressent au développement de la ville nouvelle, tel François-Xavier Ortoli, ministre de l'Équipement (1967-1968) puis de la Recherche scientifique (1969-1972). Pierre Billecocq, secrétaire d'État à l'Éducation Nationale (1969) et maire d'une commune de la Communauté urbaine de Lille (CUDL) défend la création de l'École Normale Nationale d'Apprentissage. Enfin, Norbert Ségard, fondateur en 1956 de l'Institut supérieur d'électronique du Nord (ISEN), devenu secrétaire d'État aux Postes et Télécommunications en 1976, y favorise l'implantation de cette administration. Mais ces quelques centres de recherche et de formation supérieure n'ont pas eu l'effet multiplicateur d'emplois attendu. Les explications avancées sont multiples : proximité de Paris, refus des cadres de venir dans la région, concurrence du sud de la France. S'y ajoutent le faible intérêt des industriels locaux pour la recherche et la difficulté d'attirer les industriels du Nord de l'Europe. L'argument environnemental n'a finalement pas pesé au regard de l'inachèvement de la ville et au manque de masse critique d'un pôle d'innovation embryonnaire.

Face à ces résultats décevants, les initiatives de l'EPALE se sont multipliées tous azimuts dans cette période de ralentissement économique. La ville nouvelle a tenté de répondre aux besoins du marché local et national par la diversification de l'offre de terrains et de locaux, et la poursuite des programmes de bureaux prévus. Sa situation de carrefour autoroutier métropolitain et international a sans cesse été mise en avant. L'accueil d'entreprises à l'étroit dans le tissu urbain vétuste de la métropole, le développement de la grande distribution, la captation de centres régionaux de tertiaire public ou de directions régionales d'entreprises privées ${ }^{4}$ ont permis à Villeneuve-d'Ascq d'atteindre ses objectifs en matière de création d'emplois. Finalement, le secteur tertiaire, banal ou public, l'emporte sur le tertiaire de haut niveau privé que l'on souhaitait attirer. Le secteur secondaire, qui devait constituer la moitié de l'offre d'emploi de la ville nouvelle, a une part modeste, en

4. Comme d'autres villes nouvelles, Villeneuve-d'Ascq profite de la création, par les grandes entreprises françaises, de directions régionales. Le clonage des modèles de parc d'entreprises, la proximité relationnelle entre les dirigeants, les hauts fonctionnaires et le réseau des aménageurs et urbanistes facilitent la captation de ces emplois. 


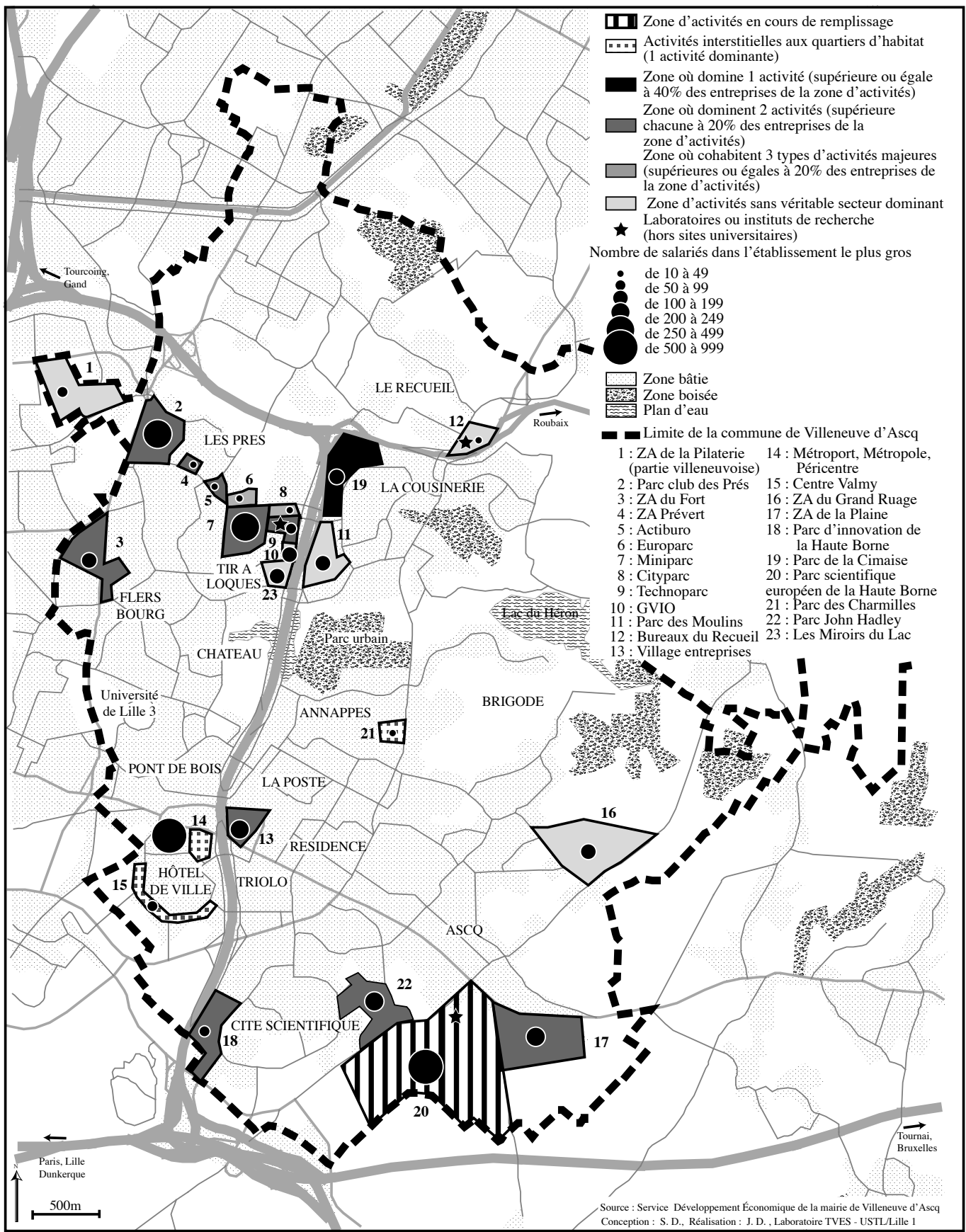

Figure 3 : Les zones d'activités de la technopole de Villeneuve-d'Ascq Business parks of Villeneuve-d'Ascq Technopole 
particulier pour les industries de pointe. Les résultats au milieu des années 1980 ne sont donc pas à la hauteur des ambitions initiales. Pourtant, à l'échelle de la métropole, la commune possède un profil original de ville tertiaire riche de ses jeunes actifs qualifiés, suscitant des jalousies au sein d'une agglomération qui peine à sortir de la crise et provoquant plusieurs tentatives d'absorption à l'initiative de Lille et/ou de Roubaix. C'est sur ce fond économique et politique complexe que le maire veut défendre l'autonomie de sa ville et lance, en 1985-1986, le projet de technopole.

\section{Créer une technopole : les moyens d'une ambition}

En 1983 s'est officiellement terminée l'aventure de la construction de la ville nouvelle. Comment trouver un nouveau souffle après cette époque d'enthousiasme fondateur? G. Caudron, le maire de Villeneuve-d'Ascq, voit dans le projet de technopole une occasion de mobiliser les forces vives de sa commune et de s'insinuer dans le jeu politique et territorial de la métropole lilloise. Le contexte politique et institutionnel du milieu des années 1980, la mobilisation des acteurs autour des enjeux économiques liés à l'innovation technologique facilitent le ralliement local des mondes de la recherche, de l'entreprise et de la politique autour de ce projet de technopole.

\section{L'ÉMERGENCE DU PROJET DE TECHNOPOLE}

Comme souvent en matière de création et d'innovation, la genèse d'une idée repose sur un mythe fondateur. Ainsi, le projet de Villeneuve-d'Ascq Technopole aurait été conçu par G. Caudron au retour d'un colloque sur les villes scientifiques organisé lors de l'exposition universelle de Tsukuba au Japon en 1985. Toutefois, les acteurs que nous avons interrogés sur les prémices de la technopole évoquent plutôt l'aboutissement d'un processus conjoint de maturation : discussions au sein de la commission des présidents d’Université sur le rôle économique et social de la recherche, échanges entre les universités de Lille 1 (USTL) et de Rennes-Atalante, voyages de techniciens de l'aménagement ou d'hommes politiques pour observer les initiatives de Sophia Antipolis et de la ZIRST de Grenoble. Avant même que le concept de technopole ait été élaboré, l'association entre recherche publique et entreprises est prônée pour contrer la concurrence des pays industriels émergents. Le lancement de la « cité de la haute technologie » du Nord-Pas-de-Calais se concrétise en 1986 par la création de l'association «Villeneuve-d’Ascq Technopole » (VAT), portée par la commune éponyme, l’Université des Sciences et Technologies, la chambre de Commerce et d'Industrie de Lille-Roubaix-Tourcoing $(\mathrm{CCI})$ et par le délégué régional à la Recherche et à la Technologie (DRRT). À l'époque, cet accord entre un maire socialiste, un président d'université communiste et le monde de l'entreprise a été perçu par certains comme une association contrenature, dans une région marquée par un affrontement historique entre patronat et syndicats. La convergence d'intérêt entre acteurs issus de mondes différents a été facilitée par des proximités politiques (le maire et le président de l'USTL), professionnelles (le DRRT est enseignant-chercheur à l'USTL) ou géographiques (le président de la CCI est PDG d'une entreprise de robotique à Villeneuve-d'Ascq).

La décision de faire de Villeneuve-d'Ascq une technopole émerge aussi dans un contexte national et régional de mutations économiques, institutionnelles et politiques qui favorise l'émergence de nouvelles pratiques, au-delà des clivages idéologiques et des équilibres de pouvoir entre les différents échelons territoriaux. C'est l'époque où le Nord-Pas-de-Calais peut envisager un nouvel avenir avec la construction du tunnel sous la Manche, l'arrivée du TGV, les premières discussions sur la création du centre d'affaires Euralille, et la mission Urba 2000 chargée de promouvoir l'usage des NTIC. À l'échelle nationale, Pierre Mauroy, premier ministre et maire de Lille, prend acte de la nouvelle donne internationale et amorce la conversion de la gauche à l'économie de marché. Ce revirement idéologique participe-t-il d'un changement de mentalité d'une partie des hommes politiques de gauche de la région? C'est sans doute un élément d'explication, au même titre que la circulation des idées entre les acteurs politiques locaux et ceux ayant acquis une stature nationale 
et gouvernementale. La décentralisation permet enfin au paradigme du développement par l’innovation technologique de se diffuser à tous les échelons territoriaux. Si l'État a dans un premier temps opéré une déconcentration régionale des structures de soutien à l'innovation, les acteurs régionaux sont incités dès 1984 à soutenir des entreprises et des laboratoires locaux dans le cadre du contrat de plan État-Région. L'idée de faire de Villeneuve-d'Ascq une technopole s’inscrit dans ce contexte d'innovations politiques et institutionnelles. L'occasion est donnée à G. Caudron de donner à sa ville une place de choix dans la conurbation, aux côtés des trois grands pôles historiques et politiques que sont Lille, Roubaix et Tourcoing.

\section{COMMENT VALORISER UN POTENTIEL TECHNOPOLITAIN?}

Au milieu des années 1980, l’image de Villeneuve-d'Ascq est celle d'une ville nouvelle inachevée, d'une ville campus, d'un faubourg de Lille. Son maire souhaite proposer à ses habitants, qu'il considère comme des pionniers, un projet plus ambitieux que celui de vivre dans une banlieue verte et tranquille. Il ambitionne aussi d'imposer sa ville comme le quatrième grand pôle urbain de la métropole lilloise, en jouant notamment sur sa position d'unique technopole au nord de Paris. Le potentiel existe : $70 \%$ de la capacité universitaire régionale, des écoles d'ingénieurs réputées, des centres de recherche de niveau national, le plus grand réseau câblé de France, un métro unique au monde (le $V_{A L}{ }^{5}$ ), des entreprises d'électronique et d'informatique qui abondent les activités de télécommunication déjà attirées dans les années 1970. Comment valoriser ce «microcosme exceptionnel dans le Nord » (Baudelle, 1984) ? L'association Villeneuve-d'Ascq Technopole a pour mission de transformer une juxtaposition spatiale de ressources technologiques en milieu technopolitain. Elle a aussi pour fonction de promouvoir la nouvelle image de la ville afin d'en conforter l'attractivité.

Dès 1987, VAT organise les «petits-déjeuners de la technopole » afin de développer la synergie entre chercheurs et entrepreneurs locaux. La participation à des salons professionnels vise à promouvoir la technopole, en particulier auprès des entreprises européennes de pointe qui pourraient, par leur implantation, accroître les ressources technopolitaines de Villeneuve-d'Ascq. Cet objectif nécessite d'élaborer une véritable politique de communication et de marketing territorial, dont la définition progressive est riche d'enseignements ${ }^{6}$. En trois ans, VAT passe d'un discours incantatoire, centré sur la seule image de marque du projet technopolitain, à une politique de valorisation des entreprises innovantes locales, des sociétés de pointe déjà implantées à Villeneuve-d'Ascq, des universités et laboratoires, des structures de soutien à la création d'entreprises et au transfert de technologie. La situation géographique de la ville, au carrefour des autoroutes vers Paris, Londres et Bruxelles, est aussi valorisée. Enfin, pour marquer sa différence, VAT met en avant la qualité du cadre de vie. Avec trois cents hectares d'espaces verts, un lac au cœur de la ville, deux golfs, des parcs d'entreprises dans la verdure, la ville vise en particulier les décideurs de l'Europe du Nord-Ouest, qui érigent l'écologie en art de vivre. Ainsi s'élabore le concept de «Technopole verte» et son logo ${ }^{7}$. Ces actions de communication sont en outre confortées par l'adhésion à l'association internationale des parcs scientifiques, puis au réseau France Technopôles. L'objectif est de s’insérer dans des réseaux scientifiques, politiques et économiques, sans lesquels le milieu technopolitain n'existe pas. La création en 1989, sur la Cité scientifique, d'un centre de conférences international équipé d'un système de vidéo-conférence et d'une antenne satellite, équipements rares à l'époque, participe de cette volonté d'insertion dans les réseaux internationaux de la recherche et de l'économie innovante. Cette mise en relation d'acteurs n'est en fait que la partie émergée de l'iceberg dans le travail de construction d'une technopole. VAT est une struc-

5. Véhicule Automatique Léger.

6. La participation, en 1989, au salon Flanders Technology International de Gand (Belgique), accueillant 250000 visiteurs professionnels, est l'acte de naissance d'une politique de communication visant à affiner le positionnement technopolitain de Villeneuve-d'Ascq.

7. « Une sphère bleue pour l'aspect technologique de la ville et une feuille verte pour le respect de l'environnement » [www. villenewvedascq.fr]. 
ture sans grands moyens, s'appuyant sur les ressources humaines du service de développement économique de la ville de Villeneuve-d'Ascq. D'autres acteurs et d'autres actions participent à l'aventure technopolitaine.

\section{DES EXPÉRIMENTATIONS INSTITUTIONNELLES AU SERVICE DE L'INNOVATION TECHNOLOGIQUE}

La création d'entreprises innovantes en relation avec des laboratoires de recherche publics est au fondement du modèle technopolitain. Pour favoriser les initiatives, la Ville et l'USTL vont initier des expérimentations institutionnelles, parfois à la limite de la légalité mais tolérées par la préfecture ou les ministères. Ainsi, dès 1984-1985, donc avant le lancement du projet de Villeneuve-d'Ascq Technopole, la mairie met à la disposition des créateurs d'entreprise des locaux municipaux vacants dispersés sur la commune, à des prix modérés. La Ferme Dupire, un ancien bâtiment agricole, devient la première pépinière publique de la commune et accueille en priorité des entreprises innovantes. À l'USTL, la location de bureaux dans des bâtiments de recherche est une pratique ponctuelle et informelle, issue de la demande de créateurs d'entreprise travaillant avec des laboratoires. Un bâtiment d'accueil finira par être construit par un promoteur privé, à la lisière du campus, mais cette pépinière semble avoir disparu quand s'est ouvert Technoval, la nouvelle pépinière de la mairie de Villeneuve-d'Ascq.

L'histoire de Technoval est significative de l'effervescence des débuts de la technopole. Tout commence en 1987 avec le souhait de l'entreprise agro-alimentaire Bonduelle de revenir dans le Nord et d'installer son siège social européen à proximité de l'université dans un cadre verdoyant. Pour G. Caudron, cette implantation est susceptible d'attirer d'autres entreprises, au moins par effet d'image. Comme la société Bonduelle souhaite s’installer dans un bâtiment provisoire proche de son siège social en construction, la ville, avec l'accord de l'Université, entreprend de rénover à grands frais et en trois mois un préfabriqué construit dans l'urgence des débuts de la Cité scientifique, mais qui accueillait encore des étudiants dans des conditions de travail déplorables. Quand Bonduelle intègre son siège social, la ville transforme le préfabriqué rénové en une pépinière d'entreprises innovantes baptisée Technoval. Des sociétés jusqu’alors hébergées à la Ferme Dupire y sont transférées. La localisation de Technoval s'avère stratégique, à proximité d'un nœud autoroutier, à la lisière du campus de l'USTL et à l'entrée de terrains universitaires cédés à la Ville pour créer le parc d'innovation de la Haute-Borne. Cette dernière opération est significative de l'esprit de collaboration institutionnelle qui porte le projet technopolitain à partir du milieu des années 1980. Des PME se sont installées sur le parc, au même titre que la météorologie nationale, l'Institut National de Recherche sur les Transports et leur Sécurité (INRETS) et le Groupement Régional Nord / Pas-de-Calais pour la Recherche dans les Transports (GRRT).

\section{L'USTL ET LES ENTREPRISES : DES CONTRATS AUX « ATELIERS DE SERVICE »}

L'USTL n'a pas attendu le lancement de la technopole pour établir des contrats entre laboratoires et industriels, sous la forme d'une relation bilatérale, informelle et quasi-confidentielle. L'implication de l'USTL dans le développement régional se traduit par l'inauguration, en 1983, de la première ligne de métro de Lille, dont le système entièrement automatisé a été conçu par l'équipe du Professeur Gabillard en collaboration avec la société Matra. C'est le fruit de dix années de coopération entre l'EPALE et la recherche publique et privée. Si l'USTL n'en a pas retiré de royalties, le prestige national et international du VAL a permis au laboratoire de son concepteur d'obtenir de nouveaux contrats. Le partenariat public/privé s'est élargi ensuite à travers la création du GRRT ${ }^{8}$ (Stachowski, 2002). Moins prestigieuse mais plus lucrative pour l'USTL fut l'invention du Nanoréseau (un réseau local d'ordinateurs) en collaboration avec Léanord, une société issue en

8. Créé en 1983, modifié en 1985, le GRRT rassemble le Conseil régional, l’USTL, l’Université de Valenciennes et du HainautCambrésis (UVHC), l'INRETS, le CNRS, les entreprises Alsthom, Matra et CIMT, la CUDL et des écoles d'ingénieurs. 
1960 de l'ISEN, institut d'électronique de la Faculté Libre des Sciences de Lille. Second fabricant français de micro-ordinateurs au début des années 1980, Léanord standardise le Nanoréseau avec le soutien de l'ANVAR et le fait breveter. Ce système est diffusé auprès des établissements d'enseignement dans le cadre du plan gouvernemental «Informatique pour tous ». Bull, dont l'usine de Villeneuve-d'Ascq assemble dès 1985 des micro-ordinateurs, est associé à la fourniture des machines pour couvrir l'ensemble du marché national (Liefooghe, 1985; Paris, 1993). La collaboration entre l'USTL et l'ISEN s'élargira, sur d'autres projets, à l'université de Valenciennes et conduira en 1992 à la création de l'Institut d'électronique et de micro-électronique du Nord (IEMN).

En dehors des contrats de recherche classiques, l’USTL expérimente au début des années 1980 des « ateliers de service » en électronique, informatique, automatisme, environnement et chimie. L'objectif est de mettre à disposition du milieu économique régional, les savoirs et savoir-faire élaborés dans les laboratoires. Ces ateliers, hébergés au sein de l'USTL, doivent s'autofinancer en travaillant sur des programmes de développement proposés par des entreprises. Chercheurs et doctorants en constituent la force de travail, mais du personnel complémentaire peut être embauché sous contrat, en fonction des projets industriels. Le Centre d'évaluation et de faisabilité économique (CEFE) est créé en 1984 pour gérer ces ateliers qui, à terme, doivent devenir des entreprises de service indépendantes. Cette expérience s'est arrêtée après une dizaine d'années, avec des résultats ambigus. D'un point de vue institutionnel, ces ateliers n'ont pas su prendre leur autonomie. Le CEFE a été dissout en 1997 mais une partie des fonctions a donné naissance à l'actuel service Relations Université-Entreprises (RUE). Du point de vue des retombées économiques régionales, les ateliers ont permis le transfert de connaissances vers de grandes entreprises, qui ont ensuite créé leurs propres services de développement et diminué en conséquence les demandes auprès de l'USTL. Quant au transfert de technologie susceptible de faire émerger d'autres secteurs d'activités dans la région, il ne fut jamais vraiment à l'ordre du jour. L'USTL a, il est vrai, conçu le projet d'un atelier de productique automobile, en collaboration avec l'Université de Valenciennes et le laboratoire de développement de la société Renault, mais ce projet n'a jamais vu le jour faute d'obtenir l'aval de Pierre Mauroy, alors premier ministre. En revanche, le succès de l'AREMI, l'atelier de service en électronique et informatique, a conforté le projet de création de l'IEMN.

Les expérimentations institutionnelles en faveur des entreprises innovantes et du développement des relations université-entreprises ont souvent anticipé la décision politique d'inscrire Villeneuved'Ascq sur la carte des technopoles françaises. L'association Villeneuve-d'Ascq Technopole a eu pour vocation d'amplifier ces initiatives, de favoriser l'émergence d'un milieu technopolitain et de développer l'attractivité de la ville autour du concept de « Technopole verte ». Quel bilan peut-on tirer de cette politique, vingt années plus tard et à l'échelle de la commune?

\section{Villeneuve-d'Ascq : une ville attractive pour la «classe créative »?}

La qualité de vie est un élément de promotion des technopoles. Attirer des entreprises de haute technologie et, surtout, la « classe créative » dont elles ont besoin (Florida, 2002) est aujourd'hui l'ambition des «villes du savoir ». L'argumentaire semble se perpétuer, au-delà des changements de dénomination. Le débat en cours sur ce qui fonde cette «qualité de vie » et sur son rôle dans l'attractivité des populations les plus qualifiées (Tremblay, 2006) fait écho à la problématique villeneuvoise des vingt dernières années. La qualité du cadre de vie, de la vie sociale et culturelle participe du discours de promotion de la technopole. Peut-on cependant évaluer l'impact de ce marketing territorial? Quelle est la part des héritages de l'opération Ville Nouvelle? La prudence s'impose pour tirer le bilan d'une action politique qui doit tenir compte des aspirations d'une population communale socialement hétérogène, ainsi que de la dynamique démographique et économique du reste de la métropole lilloise. 


\section{UN PROFIL SOCIO-PROFESSIONNEL TECHNOPOLITAIN ?}

La population active résidant à Villeneuve-d'Ascq occupe essentiellement des emplois tertiaires (tableau 1), en particulier dans les services aux entreprises. En cela, la ville s'inscrit dans le processus de tertiarisation de la métropole. Les cadres et professions intellectuelles y sont surreprésentés, à l'échelle régionale (tableau 2), de même que les professions intermédiaires, mais à un degré plus faible. À l'inverse, les ouvriers y sont sous-représentés. Ce profil est-il caractéristique d'une technopole ou est-il à rapprocher de celui d'une ville nouvelle? La spécificité de Villeneuved'Ascq, une ville nouvelle de taille moyenne de la banlieue lilloise, rend la comparaison terme à terme impossible. À Toulouse (Gregoris, 1991), on retrouve bien une structure similaire à celle de Villeneuve-d'Ascq, mais le cadre d'étude englobe toute la métropole. Dans les villes nouvelles de la région parisienne, les cadres et les professions libérales restent par contre sous-représentés, à l'exception de Saint-Quentin-en-Yvelines qui fait partie intégrante de la Technopole Sud-Île-deFrance (Davezies, 2004). Toutes choses égales par ailleurs, Villeneuve-d'Ascq semble se rapprocher du cas de Saint-Quentin-en-Yvelines, ville nouvelle et technopole.

\begin{tabular}{|l|c|c|c|c|}
\hline Secteurs & $\begin{array}{c}\text { Nord-Pas-de- } \\
\text { Calais } \\
\text { Nombre d'actifs }\end{array}$ & $\begin{array}{c}\text { Villeneuve-d'Ascq } \\
\text { Nombre d'actifs }\end{array}$ & $\begin{array}{c}\text { Nord-Pas-de- } \\
\text { Calais en } \%\end{array}$ & $\begin{array}{c}\text { Villeneuve-d'Ascq } \\
\text { en \% }\end{array}$ \\
\hline Agriculture & 34774 & 131 & $2,53 \%$ & $0,51 \%$ \\
Industrie & 290824 & 3003 & $21,20 \%$ & $11,78 \%$ \\
Construction & 76305 & 739 & $5,56 \%$ & $2,90 \%$ \\
Tertiaire & 970020 & 21610 & $70,71 \%$ & $84,80 \%$ \\
dont Commerce & 188412 & 3307 & $13,73 \%$ & $12,98 \%$ \\
$\begin{array}{l}\text { dont Services aux } \\
\text { entreprises }\end{array}$ & 150067 & 3954 & $10,94 \%$ & $15,52 \%$ \\
\hline
\end{tabular}

Tableau 1 : Population active au lieu de résidence par secteurs d'activités à Villeneuve-d'Ascq et dans le Nord-Pasde-Calais (Sources diverses : INSEE, RGP de 1999)

Region

Working Population by Branch of Industry in Villeneuve-d'Ascq and in the Nord-Pas-de-Calais

\begin{tabular}{|l|c|c|}
\hline CSP & $\begin{array}{c}\text { Nord-Pas de Calais } \\
\text { en } \%\end{array}$ & $\begin{array}{c}\text { Villeneuve-d'Ascq } \\
\text { en } \%\end{array}$ \\
\hline Agriculteurs & $1,5 \%$ & $0,1 \%$ \\
Artisans, commerçants & $5,2 \%$ & $3,3 \%$ \\
Cadres, prof. Intel. & $10,3 \%$ & $19,8 \%$ \\
Professions intermédiaires & $23,2 \%$ & $29,2 \%$ \\
Employés & $28,7 \%$ & $29,9 \%$ \\
Ouvriers & $31,0 \%$ & $17,6 \%$ \\
\hline
\end{tabular}

Tableau 2 : Les catégories socio-professionnelles (CSP) de la population active résidant à Villeneuve-d'Ascq en 1999 (Sources diverses : INSEE, RGP de 1999)

Socio-professional Groups of the Working Population living in Villeneuve-d'Ascq in 1999

La spécificité socio-professionnelle villeneuvoise est héritée d'une offre de logements diversifiée et de qualité, développée dans la période Ville Nouvelle pour répondre à une demande massive à l'échelle de la métropole. À l'époque, les HLM sont recherchées, au regard de l'état souvent insalubre des noyaux urbains anciens de l'agglomération. Le centre-ville et le Pont de Bois, presque 
exclusivement composés d'immeubles de logements collectifs, accueillent les jeunes couples. La mixité sociale de ces quartiers est réelle - à cette époque - alors que les quartiers préexistants (La Poste et La Résidence) ont une vocation plus sociale. Mais la commune a aussi pour mission d’héberger les classes moyennes (quartier des Prés, La Cousinerie) et pour ambition d'attirer des cadres par des logements individuels construits dans un écrin de verdure (opération du golf de Brigode). Favoriser la mixité sociale est un objectif de l'EPALE, repris et conforté pendant trente ans par l'équipe municipale. Aujourd'hui, Villeneuve-d'Ascq est une ville où les cadres et les professions intermédiaires sont certes surreprésentés, mais où résident aussi beaucoup de ménages modestes (47\% logent en HLM) et de nombreux étudiants.

\section{UNE « TECHNOPOLE VERTE » POUR LES CADRES OU UNE « VILLE POUR TOUS »?}

L'attractivité de Villeneuve-d'Ascq tient d'abord à l'originalité de son cadre urbain et architectural. Les formes urbaines contemporaines et les formes rurales des noyaux villageois antérieurs se mêlent en une entité nouvelle dont la qualité paysagère est aujourd'hui reconnue. La «ville verte » est le fruit d'expérimentations architecturales permises par la ville nouvelle. Le quartier du Château illustre les recherches menées autour des groupements d'habitats individuels et de la notion d'habitat intermédiaire. Fondées sur l'idée que chaque logement doit offrir une extension sous forme de terrasse, jardin ou patio, ces figures urbaines modernes mêlent ville et nature. L'urbanisme de quartier associé à ces expérimentations formelles a conduit les habitants à établir de nouveaux modes relationnels. L’offre de services de proximité (écoles, garderies, locaux associatifs) et d'équipements culturels et sportifs de qualité dans un cadre verdoyant participe de l'image positive de Villeneuve-d'Ascq dans la métropole lilloise. La «ville verte » est aussi pour partie la conséquence de l'arrêt de la ville nouvelle en 1983, et de l'abandon consécutif de nombreux programmes de logements, de bureaux et d'immeubles commerciaux. Le caractère encore rural de certains quartiers est enfin un choix politique, comme le refus de G. Caudron de laisser construire près de 3000 logements aux abords du lac du Héron. Cent hectares d'espace agricole ont ainsi été préservés, puis transformés en « réserve naturelle volontaire » à partir du milieu des années 1990.

La hausse tendancielle des prix de l'immobilier affecte aujourd'hui les espaces de qualité de la technopole et les noyaux villageois initiaux s'embourgeoisent. Les difficultés réelles des jeunes ménages villeneuvois à se loger sur la commune provoquent leur migration, ce qui a eu pour conséquence inédite une baisse de la population entre 1999 et 2005. La part des enfants de moins de 6 ans a diminué de $18 \%$ en 4 ans et c'est aujourd'hui la génération des 45-60 ans qui domine. En outre, dans une ville de faible densité où les espaces constructibles font défaut, les disparités socio-spatiales s'accentuent au détriment des quartiers en difficulté. Pour enrayer ces phénomènes, la municipalité a décidé de reprendre la construction de logements, suspendue depuis 1985. La densification de l'habitat individuel et l'accession à la propriété sont à nouveau à l'ordre du jour. Dans un contexte de prix immobiliers élevés, les résultats de cette politique sont hypothétiques. La mixité sociale voulue par l'EPALE et la municipalité entre donc en contradiction avec l'ambition de faire de Villeneuve-d'Ascq une «Technopole verte » attractive pour les populations les plus qualifiées. Cette dichotomie sociale engendre périodiquement des débats politiques locaux, notamment à l'approche des élections. Le discours technopolitain, puis la relance du projet du parc scientifique de la Haute-Borne à partir de 1996, plaident en faveur des emplois hautement qualifiés. Or, le chômage touche fortement les quartiers à dominante sociale où des problèmes de délinquance ont surgi dès les années 1990. L'équipe municipale doit constamment ajuster son discours entre promotion de la technopole et développement de l'emploi pour tous. Y a-t-il pour autant contradiction entre ces deux objectifs? Une des missions de Villeneuve-d'Ascq, comme toute ville nouvelle, est d'offrir des emplois sur place à ses habitants, afin de réduire les migrations pendulaires et d'éviter le phénomène de banlieue dortoir. Toutefois, aujourd'hui, vivre dans la ville signifie de moins en moins y travailler. Les navettes domicile/travail sont de fait élevées dans 
l'agglomération lilloise, et Villeneuve-d'Ascq est un pôle d'emploi plus dynamique que l'ensemble de l'arrondissement. La commune regroupe plus de $80 \%$ des emplois salariés privés, alors même qu'elle représente moins de $60 \%$ de la population de la zone. Quels sont les facteurs d'attractivité de la ville vis-à-vis des entreprises?

\section{LES PARCS D’ACTIVITÉS DE LA TECHNOPOLE VILLENEUVOISE : UNE OFFRE ATTRACTIVE}

Près de la moitié des entreprises installées à Villeneuve-d'Ascq dans la période Ville Nouvelle (Attia El-Hili, 1984) puis lors du lancement de la technopole (Faucret et al., 1991) sont des transferts internes à l'agglomération, Lille étant la zone de départ principal. La création d'entreprises contribue aussi à faire de Villeneuve-d'Ascq un des pôles de développement de l'agglomération lilloise. Dans la concurrence intra-métropolitaine, la ville a l'avantage de proposer aux entreprises une large gamme de produits immobiliers, et des surfaces allant de quelques centaines de mètres carrés à plus de 10 hectares d'un seul tenant (fig. 3). Les parcs d'activités créés dans les années 1970 sont dédiés soit aux entreprises de haute technologie (Tir à Loques, Recueil), soit aux entrepôts, à l'artisanat et aux activités industrielles classiques (Le Fort, La Pilaterie, le Grand Ruage). S'y ajoutent les immeubles de bureau du centre-ville en construction (photo 1) et le parc d'entreprises du Triolo inséré dans l'habitat et dédié aux services. Ce panel est complété dans la décennie suivante par une offre de bureaux innovante (photo 2). Le parc des Moulins, créé en 1980 aux abords de la voie autoroutière intra-urbaine et du parc urbain, propose une vingtaine de petits immeubles personnalisables dans un cadre de verdure. La même année, le parc club des Prés offre un produit similaire, enrichi d'une offre en services communs pour les entreprises. À partir de 1986, la zone du Tir à Loques est restructurée (Miniparc, Technoparc) et un chapelet de miniparcs s'insère dans les quartiers d'habitat entre Les Prés et le parc des Moulins. Le centreville continue de s'étoffer en immeubles de bureaux de plus grande hauteur, avec rez-de-chaussée commerciaux. Au sud de la ville, la zone d'activités de la Plaine vient compléter l'offre classique du Grand Ruage, tandis que des bureaux sont construits aux abords de la Cité scientifique, en particulier sur le parc d'innovation de la Haute-Borne.

Malgré les aléas du marché de l'immobilier dans les années 1990, Villeneuve-d'Ascq a poursuivi une politique d'offre diversifiée et de qualité, facteur d'attractivité au sein de la métropole. La vitalité actuelle de la demande en immobilier d'entreprise ${ }^{9}$ et la saturation de l'offre sur le secteur rendent le marché local très tendu, contraignant à diversifier l'offre. Le succès du Synergie park, implanté à proximité directe de la Cité Scientifique et de la Haute-Borne, correspond au marché des entreprises régionales de taille moyenne qui souhaitent établir leur siège social dans un bâtiment identifiable et personnalisé. Le nouveau parc scientifique européen de la Haute-Borne (fig. 2) offre, quant à lui, 140 hec-

Photo 1 : Immeuble de bureau de la CRAM, Quartier de l'Hôtel de ville, Villeneuve-d'Ascq (cl. I. Estienne, mars 2006)

Office Building, "Hôtel de ville" Quarter, Villeneuved'Ascq

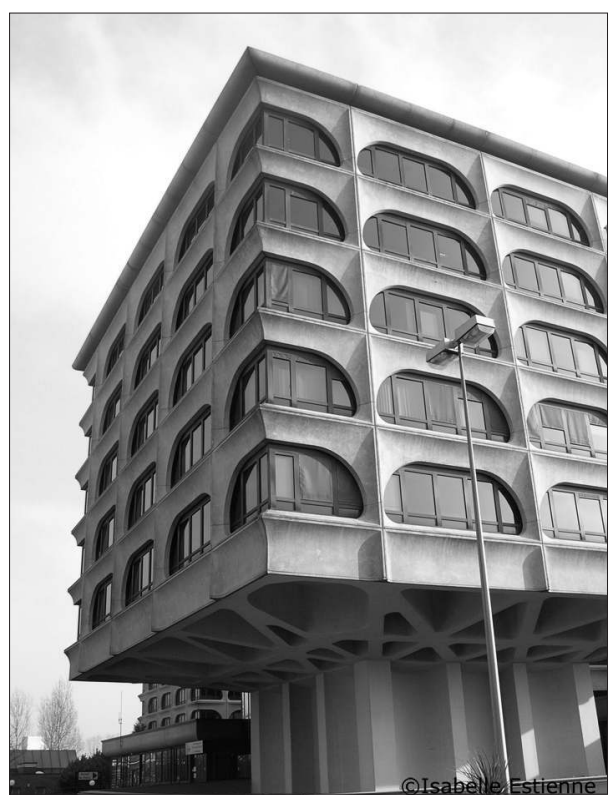

9. D’après une étude réalisée par la société Arthur Lloyd en 2001. 


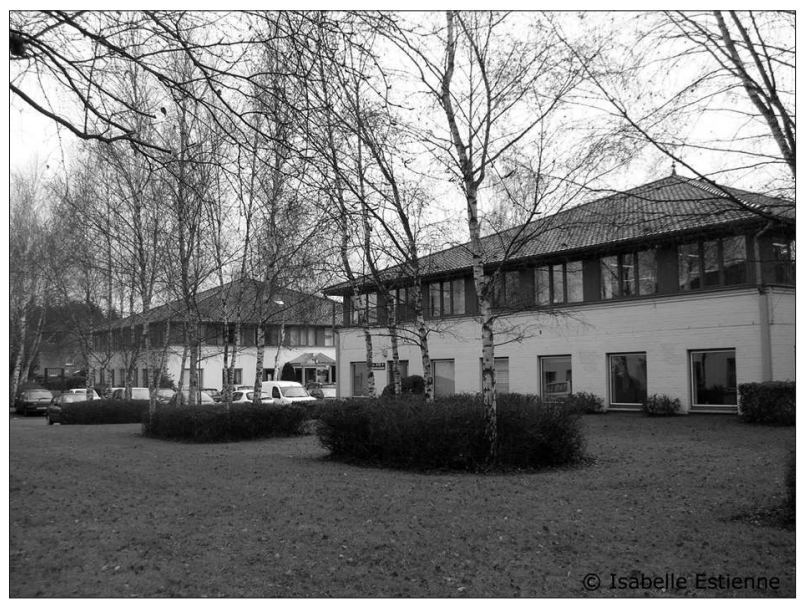

Photo 2 : Bureaux de la zone d'activités Prévert, Les Prés, Villeneuve-d'Ascq (cl. I. Estienne, mars 2006)

Business Offices, Prévert Park, Les Prés, Villeneuve-d'Ascq

tares dédiés à la construction de bureaux pour des laboratoires et des entreprises liées à la recherche. Cette option limite les implantations de sociétés privées, alors même que le marché permettrait, d'après le maire de Villeneuve-d'Ascq, de remplir le parc en deux ans. L'implantation du siège social de COFIDIS, société spécialisée dans la distribution de crédits à distance, est néanmoins significative du manque de grandes surfaces sur la métropole : le groupe Trois Suisses a obtenu d'y rassembler des bureaux dispersés dans l'agglomération lilloise. Plus de 500 emplois s'y concentrent. Bien que n'étant pas directement liée à la recherche, cette entreprise est toutefois grande utilisatrice de systèmes et technologies de l'information et de la communication (STIC).

\section{UNE VILLE ATTRACTIVE POUR LES SERVICES DE HAUT NIVEAU}

En dépit de taxes foncières et immobilières qui n’ont cessé de croître, Villeneuve-d'Ascq est un pôle d'attractivité pour les services de haut niveau, essentiellement des sociétés de «conseils et assistance » et des SSII. Ces entreprises se partagent entre les bureaux du centre-ville et les parcs d'activités qui offrent des locaux modulables dans un cadre verdoyant. Sur les seuls parcs des Moulins et des Prés, une estimation de 1990 comptabilise près de 2700 emplois, dont 45 \% d'ingénieurs ${ }^{10}$ (Faucret et al., 1991). Un bilan de la technopole réalisé à la même époque à la demande de la mairie révèle que plus de la moitié des entreprises interrogées sont indifférentes à la présence des universités et des laboratoires de recherche (Bernard, 1992). Les facteurs de localisation décisifs ont été la situation de la ville, l'offre immobilière, le cadre de vie, et très secondairement l’image de technopole. L'attractivité de Villeneuve-d'Ascq reposerait donc plus sur le cadre de vie construit par la ville nouvelle et conforté par la municipalité que sur l'enjeu technopolitain mis en exergue à partir de 1986. L'offre en immobilier de bureau est adaptée à la demande des services liés à la connaissance, dont la main-d'œuvre est sensible à l'environnement de travail. Toutefois, la « technopole verte » n'est qu'un des pôles de développement des services de haut niveau dans l'agglomération lilloise (Damette et al., 1997). Pour les SSII, cet espace périphérique de qualité est en concurrence avec une autre forme de qualité spatiale, la centralité urbaine du Grand Boulevard entre Lille et Marcq-en-Barœul (May, 2000). Le choix entre ces deux localisations dépend en partie des représentations des entrepreneurs, quant à l'image qu'ils se font du lieu « où il faut être » pour leur profession. La qualité de vie, aussi relative que puisse être cette notion, est bien un facteur d'attractivité de la main-d'œuvre très qualifiée. Villeneuve-d'Ascq est-elle pour autant 
une «ville du savoir »? Créativité et innovation sont au cœur du développement des sociétés de service de haut niveau. En 1990, un tiers des entreprises des parcs des Prés et des Moulins réinvestit effectivement une part du chiffre d'affaires dans la Recherche et Développement (R\&D), et $45 \%$ des PME interrogées accueillent des stagiaires des universités et écoles d'ingénieurs de toute la métropole (Faucret et al., 1991). Mais seules $11 \%$ des sociétés ont des relations de recherche avec les laboratoires de Villeneuve-d'Ascq. Un travail de terrain d'envergure reste à effectuer pour confirmer ces hypothèses et en vérifier l'actualité. Mais quelle est la pertinence d'une telle étude à l'échelle de Villeneuve-d'Ascq, quand la dynamique technopolitaine se diffuse au moins à l'échelle de la métropole, sinon à celle de la région?

\section{Les échelles de la dynamique technopolitaine : réseaux et gouvernance}

La dynamique technopolitaine repose sur le développement de technopôles, mais aussi sur la mise en réseau des compétences territoriales en matière de recherche, d'innovation et de création d'entreprise. Des politiques visent souvent à conforter, voire initier, cette dynamique. L'ambition de Villeneuve-d'Ascq était de devenir une technopole, une ville dont le dynamisme technopolitain aurait été au service du développement de la métropole et de la région. Qu'en est-il à l'heure de la globalisation et de la métropolisation? Les technopôles se multiplient sur le territoire de la métropole lilloise et les réseaux technopolitains se tissent à l'échelle de la région. La gouvernance technopolitaine en est de plus en plus complexe.

\section{UNE DYNAMIQUE ÉCONOMIQUE TECHNOPOLITAINE À VILLENEUVE-D’ASCQ?}

La dynamique technopolitaine repose sur un enchaînement idéal : " fertilisation croisée » entre recherche et monde économique, création d'entreprises innovantes, émergence d'activités de haute technologie, développement d'un territoire. À Villeneuve-d'Ascq, les activités de pointe implantées dès la période Ville Nouvelle n'ont pas engendré de développement cumulatif d'industries de haute technologie, quelles que soient les classifications utilisées pour définir cette dernière. Des seize sociétés brandies comme des trophées lors du lancement de la technopole, il n'en reste que trois en 2005 : deux PME locales et l'entreprise Bonduelle. Cette société a certes une politique d'innovation de produits et de procédés à forte composante technologique, mais l'agro-alimentaire n'a jamais été considéré comme une industrie de haute technologie. Quant aux grandes sociétés d'électronique et d'informatique, leurs antennes régionales - souvent de simples établissements commerciaux - ont fermé dans les années 1990. La disparition des 300 emplois de la chaîne de montage d'ordinateurs Bull est le point d'orgue de cette hécatombe. Pour ces sociétés, Villeneuved'Ascq n'était donc qu'une opportunité de localisation à une époque donnée, et non un territoire riche en ressources technologiques favorables à l'ancrage des entreprises de haute technologie. Aujourd'hui, quelques sociétés industrielles appuient leur croissance sur de la R\&D, à l'exemple des laboratoires pharmaceutiques Boiron (tableau 3). Mais ce tissu industriel n'a pas la masse critique pour engendrer un développement cumulatif.

Pour autant, Villeneuve-d'Ascq reste depuis vingt ans un pôle de création nette d'emplois et d'entreprises. Une dynamique de création/disparition entretient un renouvellement des activités de haute technologie, à condition cette fois d'adopter une définition élargie aux SSII, aux sociétés de services scientifiques, aux cabinets de conseil en gestion et organisation (Fache, 2002). Depuis les années 1980, les services aux entreprises de haut niveau jouent un rôle essentiel dans la résilience des systèmes productifs par l'innovation (Liefooghe, 2002). Leur développement sur Villeneuve-d'Ascq se traduit par une domination des services en informatique (tableau 4). À l'exception de quelques PME de 50 à 250 personnes, ce sont surtout des micro-établissements. Cette concentration spatiale des SSII n'est-elle que le fruit d'une offre immobilière adéquate? On peut émettre l'hypothèse d'une dynamique technopolitaine liée à la pépinière Technoval, qui 
accueille dès 1989 une antenne du Pôle Productique Régional et du réseau CAO, programmes régionaux mettant à disposition des PME/PMI les compétences en informatique et électronique des écoles d'ingénieurs et des universités (Bruhat, 1990). L'USTL renforce ce pôle en y transférant l'AREMI (atelier de service en informatique) et le CEFE (gestion des synergies entre l'université et les entreprises). En quinze ans, près de 120 entreprises ont bénéficié des services de Techno$\mathrm{val}^{11}$. Ces PME innovantes n'en sont pas pour autant liées à la recherche. D’une part, le marché régional des produits et services de haute technologie est faible; d'autre part, le Centre d'Innovation et de Transfert de Technologie du Nord, créé entre Lille et Villeneuve-d'Ascq à l'initiative du département, capte les dossiers les plus porteurs. L'USTL participe aussi de la dynamique du CITTN, par une implication financière dans la SEM qui le porte. De 1988 à 2005, 111 projets, totalisant 384 emplois, ont été portés par le CITTN devenu Ruche Technologique du Nord en $1993^{12}$. L'impact territorial de ces deux pépinières à «vocation technologique » est cependant mal connu. Aucune étude n'a dénombré à ce jour les emplois créés par les entreprises après leur sortie de pépinière, ni établi leur répartition géographique. Les SSII localisées à Villeneuve-d’Ascq n'ont pas toutes émergé des pépinières ou autres structures d'interface avec la recherche publique. Elles sont plutôt le fruit d'une mutation de l'appareil productif régional engagée dans les années 1980. La diffusion des technologies liées à l'informatique repose en effet sur la création cumulative de structures d'envergure régionale, telles le salon Applica en $1988^{13}$ (salon des applications de l'informatique), Infonord en 1995 (pôle des technologies et sciences de l'information), DéclicNet en 1998 (centre de ressources e-business) et Digiport en 2001 (centre de services TIC). De la productique à l'e-business, vingt années de politiques publiques partenariales ont favorisé l'émergence d'un marché régional et métropolitain dans la grappe technologique liée à l'informatique. Villeneuve-d'Ascq n'est qu'un des espaces privilégiés d’implantation des SSII dans la métropole, et la dynamique technopolitaine, quand elle existe, dépasse largement le cadre communal. Mais la vocation initiale de VAT n'était-elle pas justement de se mettre au service du développement de la métropole et de la région?

\begin{tabular}{|l|c|c|}
\hline Type d'industrie & $\begin{array}{c}\text { Nombre } \\
\text { d'établissements }\end{array}$ & $\begin{array}{c}\text { Effectifs } \\
\text { (estim. 2004*) }\end{array}$ \\
\hline Industrie électrique de haute technologie & 2 & 30 \\
Industrie médicale de haute technologie & 1 & 75 \\
Industrie pharmaceutique & 2 & 20 \\
Fabrication d'instrumentation scientifique et & 2 & 15 \\
technique & 1 & 23 \\
Fabrication d'instrumentation optique et lunettes & 2 & 164 \\
Fabrication d'ordinateurs & 10 & \\
\hline TOTAL & & \\
\hline
\end{tabular}

Tableau 3 : Les industries de haute technologie à Villeneuve-d'Ascq (Sources diverses : Service Développement Économique de la ville de Villeneuve-d'Ascq) (*effectifs estimés à partir des données par classe [centre de classe])

High Technology Mamufacturing Industry in Villeneuve-d'Ascq président de la CCI en 1986 et membre fondateur de Villeneuve-d’Ascq Technopole. 


\begin{tabular}{|l|c|c|}
\hline Type d'activité & $\begin{array}{c}\text { Nombre } \\
\text { d'établissements }\end{array}$ & $\begin{array}{c}\text { Effectifs } \\
\text { (estim. 2004*) }\end{array}$ \\
\hline $\begin{array}{l}\text { Activités informatiques : conseils en systèmes } \\
\text { informatiques, réalisation de logiciels (721Z, } \\
722 \mathrm{~A}, 722 \mathrm{C})\end{array}$ & 67 & 1419 \\
\hline $\begin{array}{l}\text { Services juridiques, comptables, conseil pour les } \\
\text { affaires et la gestion (741A, 741C, 741G) }\end{array}$ & 62 & 318 \\
\hline $\begin{array}{l}\text { Architecture, ingénierie, contrôle : architectes, } \\
\text { géomètres, ingénierie, études techniques (742A, } \\
742 \mathrm{~B}, 742 \mathrm{C})\end{array}$ & 46 & 434 \\
\hline $\begin{array}{l}\text { Publicité et études de marchés : agences conseils } \\
\text { en publicité (744B) }\end{array}$ & 16 & 39 \\
\hline TOTAL & 191 & 2210 \\
\hline
\end{tabular}

Tableau 4 : Les services de haut niveau à Villeneuve-d'Ascq (Sources diverses : Service Développement Économique de la ville de Villeneuve-d'Ascq) (* effectifs estimés à partir des données par classe [centre de classe])

Advanced Producer Services in Villeneuve-d'Ascq

\section{LA DIMENSION RÉGIONALE DES SYNERGIES TECHNOPOLITAINES}

Devenir le moteur de la reconversion régionale par l'innovation : le défi à relever était considérable pour Villeneuve-d'Ascq Technopole, compte tenu des handicaps du Nord-Pas-de-Calais. La position frontalière a longtemps expliqué l'absence d'implantations technologiques stratégiques dans une région où le plein-emploi a en outre masqué l'exigence de diversification industrielle jusqu’à la fin des années 1960. La crise du milieu des années 1970 a frappé les activités textiles et sidérurgiques au moment où elles amorçaient la modernisation de leur structure de production. VAT, avec ses faibles moyens, ne pouvait seule porter les efforts nécessaires de structuration de la recherche régionale et de soutien au transfert de technologie. Les industriels du Nord-Pas-deCalais ont encore aujourd'hui une culture de modernisation pragmatique de leur entreprise et n'ont pas l'habitude de travailler en collaboration avec des centres de recherche publics. Le nombre des chercheurs à temps plein de la recherche privée reste en deçà de la moyenne nationale, ainsi d'ailleurs que celui des établissements publics scientifiques et techniques (EPST) comme le CNRS, l'INRA et l'INSERM. Cette double faiblesse régionale porte préjudice aux collaborations entre recherche publique et entreprises, deux mondes qui suivent leur propre logique de développement dans une méconnaissance réciproque. Pour palier l’insuffisance de la recherche publique régionale, les contrats de plan Etat-Région ont eu pour priorité le redéploiement national des EPST vers le Nord-Pas-de-Calais. Cette politique a permis une augmentation de $50 \%$ des effectifs liés à la recherche entre 1990 et 1999, contre $30 \%$ en moyenne nationale (INSEE NordPas-de-Calais, 2005). Néanmoins, la région garde un handicap dans la compétition inter-régionale pour l'implantation des EPST. Le Nord-Pas-de-Calais s'était par exemple porté candidat pour accueillir à Villeneuve-d'Ascq le futur synchrotron optimisé pour l'émission intense de la lumière (projet SOLEIL). Par décision gouvernementale, l'équipement dont avait besoin la région pour être identifiée comme pôle scientifique et enclencher une dynamique de développement économique ne s'installera pas à Villeneuve-d'Ascq, mais à Orsay dans la région parisienne. En compensation, Villeneuve-d'Ascq accueillera en 2006 deux grands instituts de recherche, l'IRCICA et l'IRI ${ }^{14}$. Mais quel rôle pourra jouer l'IRI, par exemple, face à la concurrence du « campus de recherche »

14. IRCICA : Institut de recherche sur les composants logiciels et matériels pour l'information et la communication avancée. IRI : Institut de recherche interdisciplinaire à l'interface entre la physique, la chimie, la biologie. 
de Saclay, concentration de chercheurs de haut niveau qui mise aussi sur l'interdisciplinarité entre physique, chimie et biologie?

Face au poids de la recherche en région parisienne, le Nord-Pas-de-Calais n'a eu d'autre choix que de favoriser la mise en réseau des laboratoires de recherche régionaux et de les structurer en pôles de compétence de dimension nationale, voire internationale. L'objectif est d'atteindre une masse critique de chercheurs et de financements pour favoriser les synergies, dans les domaines des transports terrestres, de la biologie-santé-nutrition, des STIC, de la chimie et matériaux, ou de l'environnement. Depuis près de quinze ans, ces pôles de recherche travaillent avec de grandes entreprises, souvent non régionales, et valorisent leurs découvertes par le dépôt de brevets et la cession de licences (INSEE Nord-Pas-de-Calais, 2002). La Région a ainsi soutenu la création de l'IEMN, né en 1992 de la collaboration entre le CNRS, l'USTL, l'Université de Valenciennes et l'ISEN. Le bâtiment phare de ce réseau de chercheurs en micro-électronique et nanotechnologies est un centre de recherche d'architecture contemporaine construit à l'entrée sud de la Cité scientifique de Villeneuve-d'Ascq. Vingt millions d'euros ont été investis dans des équipements performants pour amplifier le rayonnement national et international de la recherche régionale en électronique. Au-delà des relations classiques entre laboratoires et grands groupes industriels, l'institut participe au développement régional par le biais d'IEMN-Transfert. Cette structure héberge des entreprises innovantes et propose ses compétences aux PME régionales. Elle anime enfin les «Clubs Pionniers de l'Électronique » en collaboration avec Électropôle, le pôle professionnel régional des entreprises en électronique.

Les efforts de structuration de la recherche et le soutien au transfert de technologie trouvent leur aboutissement dans les «pôles de compétitivité » labellisés par l'État en 2005, dans les domaines des transports terrestres, de la santé, des textiles techniques et innovants, des matériaux à usage domestique, de la filière des produits aquatiques, de la grande distribution et de la vente à distance. Les pôles ont pour fonction d'accélérer la mutation de secteurs d'activités dits traditionnels. Le renforcement des synergies entre centres de recherche et entreprises vise à réaliser des sauts technologiques créateurs de nouvelles niches de marché. L'IRCICA, l'IRI et l'INRIA FUTURS ${ }^{15}$, implantés dès 2006 sur le parc scientifique européen de la Haute-Borne à Villeneuve-d'Ascq, auront pour rôle de susciter des travaux interdisciplinaires applicables aux transports, à la santé et aux nouvelles formes de commerce. Les entreprises régionales parviendront-elles à valoriser les résultats de ces équipes très spécialisées? Pour les y inciter, sont prévus des exonérations fiscales et des allègements de charges dans le cadre des "pôles de compétitivité ». La délimitation des « zones de R\&D » qui conditionnent ces aides montre que la métropole lilloise participe à cinq des six pôles labellisés : l'essentiel du potentiel de recherche publique y est concentré. Les projets en cours risquent de renforcer la métropolisation de la dynamique technopolitaine régionale.

\section{DE VILLENEUVE D’ASCQ TECHNOPOLE A LA TECHNOPOLE MULTIPOLAIRE}

La problématique de développement de la métropole lilloise change à l'aube des années 1990, dans la perspective de l'ouverture du marché européen, de la construction du tunnel sous la Manche, et en lien avec le processus de métropolisation. Euralille, quartier d'affaires au croisement des lignes TGV Paris-Londres/Paris-Bruxelles, est le signe urbain de cette « bifurcation métropolitaine », transfrontalière voire européenne (Paris et Stevens, 2000). Le projet Euralille ne pouvant être porté par la seule ville de Lille, Pierre Mauroy obtient un consensus communautaire pour le financement de ce projet. Fin 1988, la charte des « grands maires » (Lille, Roubaix, Tourcoing, Villeneuve-d'Ascq, Mons-en-Barœul) prévoit la carte des futurs " pôles d'excellence métropolitains » accordés en échange de ce soutien (fig. 1). Euralille doit être connecté par fibre optique à l'Eurotéléport de Roubaix (inauguré en 1994). Eurasanté doit devenir un pôle d'entreprises innovantes autour du centre hospitalier régional. Le projet de zone d'activités de la Haute-Borne,

15. INRIA FUTURS : Institut de recherche en informatique et automatique. 
déjà programmé par la ville nouvelle, est relancé avec un statut de site technologique à vocation européenne. Les contours d'une technopole multipolaire à l'échelle de la métropole se dessinent au début des années 1990. À la même époque, une mission portée par VAT et Euralille, et animée par le président de l'USTL (Dubrulle, 1993), étudie la faisabilité d'une technopole régionale en réseau. Les domaines de spécialisation technologique du Nord-Pas-de-Calais y sont définis. La création de «clubs technopolitains » et de «zones technopolitaines potentielles » est proposée pour intégrer chacun des territoires régionaux à la dynamique technopolitaine. Dix ans plus tard, ce projet novateur est tombé dans l'oubli.

Les « pôles d'excellence » de la métropole lilloise constituent donc aujourd'hui l'offre régionale en sites technopolitains à fort potentiel, qu'il s'agit de remplir. Cette gamme de parcs aux profils technologiques spécialisés permet à Lille Métropole de se positionner dans la course à l'implantation d'établissements publics scientifiques et techniques. Cette stratégie pourrait donner à la région une masse critique de chercheurs et scientifiques qui lui fait encore défaut. Mais devant la concentration des activités de recherche sur les sites scientifiques français les plus prestigieux, le risque est réel de voir la métropole lilloise reléguée aux fonctions administrative des EPST, aux emplois essentiellement logistiques et informatiques. En dépit de l'amélioration du cadre de vie et d'une offre culturelle croissante, l'attractivité de l'agglomération lilloise sur les cadres dirigeants et les emplois très qualifiés reste encore faible. Lille est en concurrence avec d'autres métropoles régionales, mais aussi européennes. Ainsi en 1990, un projet de « cité de l'électronique » devant accueillir 200 chercheurs a mis en concurrence Villeneuve-d'Ascq, Amsterdam et Louvain (Belgique). La métropole lilloise n'a pas su le capter (Faucret et al., 1991). Le développement métropolitain suppose donc une dynamique endogène forte pouvant s'appuyer sur une articulation efficiente entre des acteurs technopolitains multiples et géographiquement dispersés.

\section{VERS UNE GOUVERNANCE MÉTROPOLITAINE DE LA DYNAMIQUE TECHNOPOLITAINE}

La multiplication des pôles technopolitains à l'échelle de la métropole pose un problème de gouvernance, et en premier lieu en matière d'aménagement. L'histoire de la zone de la HauteBorne à Villeneuve-d'Ascq est représentative de la concurrence politique entre les grandes villes de l'agglomération lilloise, et de ses conséquences en termes de priorités d'aménagement urbain dans un contexte d'aléas de la croissance économique. Conçue par l'EPALE comme une zone d'activités, la Haute-Borne n'est toujours pas aménagée à la fin des années 1980. Inscrite en 1988 sur la carte des pôles d'excellence métropolitains, il faut attendre 1996 pour que la Communauté urbaine de Lille mette le parc européen de la Haute-Borne au rang de ses priorités. Le site est alors choisi par la Région pour porter la candidature du Nord-Pas-de-Calais à l'accueil du projet SOLEIL. Politiques, universitaires et monde de l'entreprise font corps pour plaider la cause du site villeneuvois auprès du gouvernement français. La déception est au rendez-vous en 1999. Le parc scientifique européen de la Haute-Borne se construit donc sans SOLEIL, mais en réaffirmant sa vocation d'accueil d'activités liées à la recherche.

La question de la gouvernance technopolitaine se pose aussi et surtout en termes d'animation, de communication et de réseau. Ces fonctions étaient remplies par Villeneuve-d’Ascq Technopole depuis 1986. Mais cette association n'a plus la légitimité et les moyens financiers nécessaires pour assumer le portage d'une technopole multipolaire. En 1994, VAT devient Technopole Lille Métropole (TLM), soutenue par la CUDL, les universités, les grandes écoles et les structures représentatives du monde économique. TLM a pour mission d'optimiser le maillage des compétences et de monter des réseaux actifs à toutes les échelles, c'est-à-dire de conforter la fonction technopolitaine de la métropole lilloise (Stevens, 1995). Mais TLM partage ce rôle avec le GIE Eurasanté, qui porte tout à la fois une structure d'animation et de conseil, le parc dédié aux activités de biologie-santé et son bio-incubateur. De son côté, TLM porte Digiport, agence de développement des TIC récemment transférée de la Cité scientifique à Euratechnologie, et CIEL, le centre d'innovation et d'échanges de Lille, opérationnel depuis 2005 sur le parc scientifique 
européen de la Haute-Borne. La vocation de CIEL est d'animer les échanges entre universités et entreprises. Son originalité est aussi de rassembler dans un bâtiment unique des équipes compétentes au service des porteurs de projets innovants (tableau 5). Ce regroupement spatial de structures auparavant dispersées sur la métropole vise à développer les synergies entre acteurs, et permet à CIEL de jouer le rôle de vitrine d'une gouvernance technopolitaine qui se construit pas à pas depuis dix ans. CIEL a aussi une portée régionale, comme en atteste la présence du MITI Incubateur Nord-Pas-de-Calais. Créé à la faveur de la loi sur l'innovation de 1999, cette structure détecte et accompagne les projets technologiques. Mais à l'inverse de nombre d'incubateurs, le MITI n'héberge aucune entreprise. Il s'appuie pour cela sur le réseau d'incubateurs, de ruches et de pépinières déjà opérationnel dans la région (fig. 4). Le MITI est en effet devenu la tête de réseau des compétences développées depuis vingt ans dans les territoires du Nord-Pas-de-Calais, en matière de soutien à l'innovation et à la création d'entreprises innovantes. Il a aussi comme partenaires les universités régionales.

\begin{tabular}{|l|l|}
\hline Structures hébergées dans le bâtiment CIEL & Vocation de la structure \\
\hline $\begin{array}{l}\text { SAEM du parc scientifique européen de la Haute- } \\
\text { Borne }\end{array}$ & $\begin{array}{l}\text { Gestion du parc d'activités de la Haute-Borne } \\
\text { Accueil des entreprises liées à la recherche }\end{array}$ \\
\hline Équipe CIEL & $\begin{array}{l}\text { Équipe d'animation des relations entre les } \\
\text { universités et les entreprises }\end{array}$ \\
\hline Maison de l'entreprenariat & $\begin{array}{l}\text { Sensibilisation des étudiants à la création } \\
\text { d'entreprise }\end{array}$ \\
\hline Cré'Innov & Annexe du pré-incubateur CréInnov de l'USTL \\
\hline MITI Incubateur Nord-Pas-de-Calais & $\begin{array}{l}\text { Incubateur « virtuel » du Nord-Pas-de-Calais : pas } \\
\text { d'hébergement des entreprises }\end{array}$ \\
\hline Ruche Technologique du Nord & $\begin{array}{l}\text { Annexe de la Ruche Technologique du Nord sise } \\
\text { entre Villeneuve-d'Ascq et Lille }\end{array}$ \\
\hline Club Entreprise Lille Métropole Est & $\begin{array}{l}\text { Club des entreprises du versant est de la } \\
\text { métropole lilloise }\end{array}$ \\
\hline
\end{tabular}

Tableau 5 : CIEL, le Centre d'Innovation et d'Échange de Lille Métropole. Parc scientifique européen de la HauteBorne (Villeneuve-d'Ascq)

Parc (Villeneuve-d'Ascq)

CIEL, the Centre for Innovation of the Lille metropolitan Area. Haute-Borne European Science

\section{LES UNIVERSITÉS ET LA GOUVERNANCE TECHNOPOLITAINE}

L’implication des universitaires dans la gouvernance technopolitaine n'est pas négligeable. Membre de TLM, l'USTL participe à diverses structures d'animation technopolitaine (tableau 6). Acteur à part entière du développement territorial, l’université de Lille 1 est partie prenante de nombreux groupements de recherche et pôles de compétitivité régionaux. Son engagement institutionnel et/ ou financier dans le soutien à la création d'entreprise, à l'innovation et au transfert technologique s'opère aussi à travers des associations ou des structures en réseau. Sa participation financière est particulièrement significative dans le fonds régional d'amorçage INOVAM, chargé de renforcer le capital des jeunes entreprises liées à la recherche. Le rôle technopolitain de l'USTL s'exerce enfin à travers l'engagement d'une partie de son personnel, à titre institutionnel ou individuel. Présidents d'université ou enseignants-chercheurs s'impliquent dans le montage de programmes territoriaux liés à la recherche (Nord-Pas-de-Calais Technopole, projet Soleil par exemple), dans la structuration de la recherche régionale (Conseil régional, DRRT) et dans les groupes de prospective sur la métropole ou la région. Certains vont jusqu’à briguer des mandats électoraux, qui 
leur permettent parfois d'agir sur l'avenir scientifique et technologique des territoires. Au-delà de l'exemple de l'USTL, c'est en fait l'ensemble des universités lilloises et régionales qui participent désormais de la gouvernance technopolitaine.

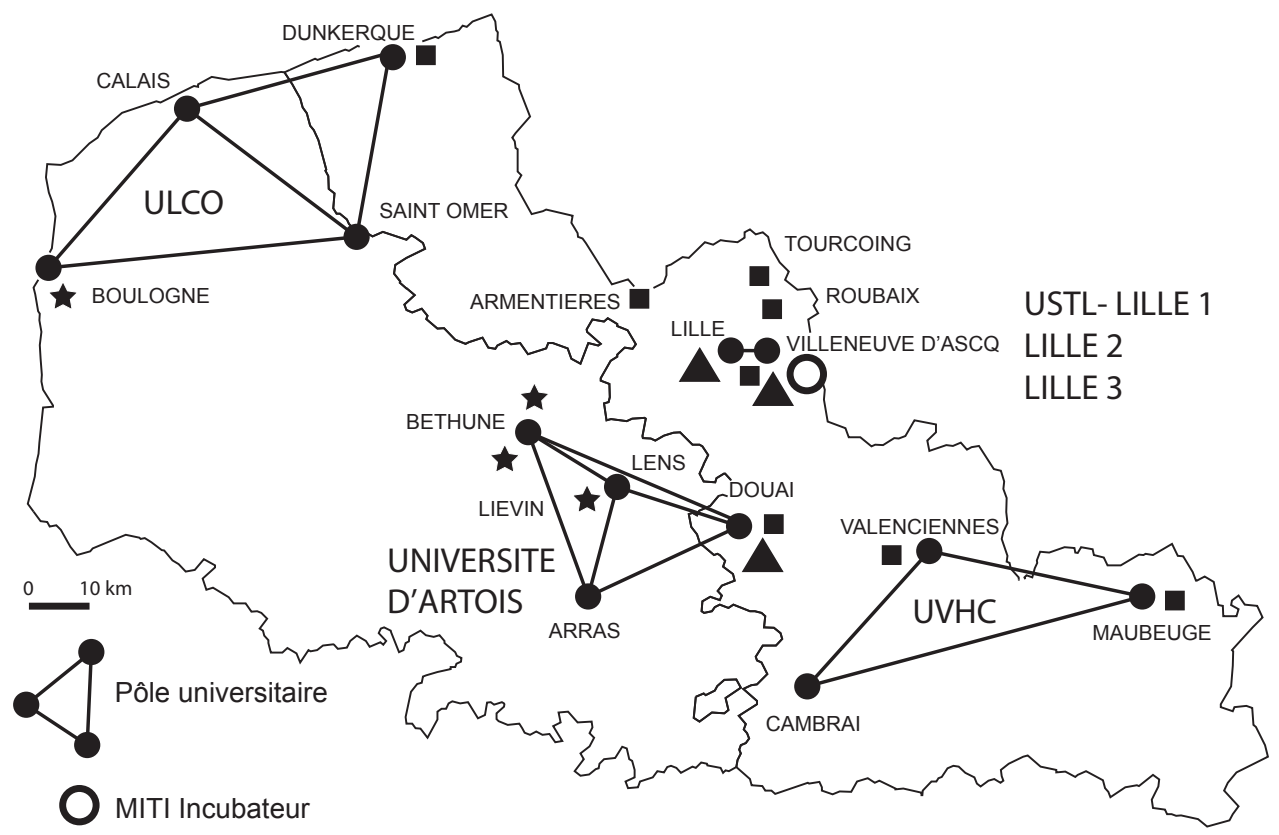

Hébergement de projets en incubation - Mise en relation avec des laboratoires

Apui - Ecole des Mines - Douai

Centre F. Kuhlman - ESC - Lille

Cré'Innov - USTL - Villeneuve d'Ascq

\section{Hébergement de jeunes entreprises}

- Réseau des Ruches du Nord (Centres Européens d'Entreprise et d'Innovation)

$\star$ Réseau d'ADITEC - Pas-de-Calais (Centres Européens d'Entreprise et d'Innovation)

Réalisation : C. Liefooghe, Laboratoire TVES, USTL/Lille 1, 2006

D'après : www.miti.fr

Figure 4 : Le MITI Incubateur Nord-Pas-de-Calais, un fonctionnement en réseau

The Partners of the MITI Incubator of Nord-Pas-de-Calais Region

Le changement d'échelle du fait technopolitain sur la métropole lilloise a modifié la gouvernance d'une technopole fonctionnant plus que jamais en réseau. Toutefois, des reconfigurations sont en cours, en réponse aux appels d'offres de l'agence nationale de la Recherche et de la DIACT (anciennement DATAR). Les pôles de compétitivité et les futures aires de coopération métropolitaine sont susceptibles d'élargir à la région la dynamique technopolitaine, mais aussi d'en rendre plus complexe la gouvernance par la multiplication, voire la superposition, des structures. Il n'est pas non plus certain que cette nouvelle donne puisse freiner la métropolisation croissante du potentiel de recherche, d'innovation et de création d'entreprise. 


\begin{tabular}{|l|l|}
\hline Type d'implication & $\begin{array}{l}\text { Exemples de structures dans lesquelles } \\
\text { s'implique l'USTL }\end{array}$ \\
\hline Soutien à la création d'entreprise & $\begin{array}{l}\text { - Réseau des ruches du département du Nord } \\
\text { - Pépinière Technoval - Villeneuve-d'Ascq } \\
\text { - MITI Incubateur Nord-Pas-de-Calais }\end{array}$ \\
\hline $\begin{array}{l}\text { Soutien à l'innovation dans les } \\
\text { entreprises }\end{array}$ & $\begin{array}{l}\text { - Contrats de recherche avec des entreprises } \\
\text { - Réseau de Développement Technologique des PME } \\
\text { - Sup'Investissement 59/62, société actionnaire du Fonds } \\
\text { Régional d'Amorçage INOVAM } \\
\text { - Pôles de compétitivité }\end{array}$ \\
\hline Animation technopolitaine & $\begin{array}{l}\text { - Technopole Lille Métropole } \\
\text { - Eurasanté } \\
\text { - SAEM du parc scientifique européen de la Haute-Borne } \\
\text { - Club Entreprise Lille Métropole Est }\end{array}$ \\
\hline
\end{tabular}

Tableau 6 : Implication institutionnelle et/ou financière de l’Université des Sciences et Technologies de Lille dans la dynamique technopolitaine (Sources diverses : USTL, Service Relations Université Entreprises, CA du 08 avril 2005)

Institutional and/or financial Involvement of the Lille University of Sciences in the technopolitan Dynamics

\section{Conclusion}

Le projet «Villeneuve-d'Ascq Technopole » a pour origine l'ambition politique d'un maire de faire de sa ville nouvelle le quatrième grand pôle urbain de l'agglomération lilloise. Le marketing territorial développé autour de la notion de «technopole verte » s'appuie sur les choix architecturaux et urbanistiques de la période Ville Nouvelle, qui ont doté la commune d'un environnement naturel recherché dans lequel s'insèrent logements et activités. La qualité de vie et des conditions de travail sont assurément un atout pour Villeneuve-d'Ascq dans la redistribution intra-métropolitaine des populations et des activités. Cette qualité urbaine et paysagère est aussi un facteur d'attractivité vis-à-vis des entreprises de haute technologie et des populations les plus qualifiées. La construction d'un véritable milieu technopolitain, par contre, se heurte à la faible croissance économique et à l'inertie du système économique local. Les entreprises dites de haute technologie implantées dans les années 1970-1980 n’ont pas joué le rôle moteur qu'on pouvait en attendre. L'université s'ouvre de plus en plus au monde économique, mais l'industrie régionale se modernise par le biais des ingénieurs et reste souvent hermétique au monde de la recherche publique. Les nombreuses sociétés de services avancés implantées sur Villeneuve-d'Ascq constituent certes un terreau pour le développement d'un milieu innovateur, mais l'espace technopolitain qui devait se construire en profitant des acquis de la période Ville Nouvelle reste à l'état de potentiel jusque dans les années 1990. En fait, l'ouverture européenne et le processus de métropolisation font basculer la problématique technopolitaine. L'ambition de Villeneuve-d'Ascq d'être la technopole régionale au service de la reconversion du Nord-Pas-de-Calais se heurte à l'aspiration métropolitaine de l'agglomération lilloise. La dynamique technopolitaine se déploie désormais à cette échelle, à travers le choix de cinq «pôles d'excellence » spécialisés. Une technopole multipolaire se dessine, qui nécessite une redéfinition de la gouvernance technopolitaine. Les réseaux de transfert technologique, d'innovation et de création d'entreprise se structurent au moins à l'échelle métropolitaine, sinon à l'échelle régionale, et les universités prennent une part active à la gouvernance de cette métropole technopolitaine en devenir. Paradoxalement, alors que Villeneuve-d'Ascq n'est plus le centre de gravité de la technopole, le parc scientifique européen de la Haute-Borne dote enfin la commune d'un technopôle, outil d'aménagement et de développement utilisé il y a plus de vingt ans par d'autres villes françaises. Les cinq parcs scientifiques ou technologiques de la métropole lilloise parviendront-ils cette fois à engendrer une dynamique technopolitaine 
porteuse d'un développement auto-entretenu? La politique des «pôles de compétitivité » peut abonder dans ce sens. Ces outils de développement sont censés accélérer la mutation du système productif du Nord-Pas-de-Calais. Ils peuvent aussi conforter la métropolisation des populations les plus créatives, et celle des fonctions et des activités technopolitaines. Mais peut-être faut-il d'abord renforcer le foyer d'innovation que devrait être une métropole, pour en espérer ensuite une diffusion du développement technopolitain à l'échelle du Nord-Pas-de-Calais.

\section{Bibliographie}

Anonyme, 1969. - Construire une métropole, Livre Blanc, Métropole Nord, Lille, Agence d'urbanisme de la Communauté urbaine de Lille, 175 p.

AtTia EL-HiLi (N.), 1984. - Ville nowvelle et planification du développement. Les zones d'activités de Villeneuved’Ascq, Mémoire de DEA de géographie, USTL/Université de Lille 1, 112 p.

BARLeS (S.), 2005. - Hydrologie et paysages urbains en villes nouvelles, Paris, Rapport pour le ministère de l'Équipement, $118 \mathrm{p}$.

Baudelle (G.), 1984. - Villeneuve-d'Ascq, ville nouvelle. Un exemple d'urbanisme concerté, Paris, CEP Éditions - Éditions du Moniteur, avec le concours de l'EPALE, 198 p.

Bernard (J.-M.), 1992. - Villenewve-d'Ascq Technopole. Analyse et évaluation d'un technopôle, Ville de Villeneuved'Ascq, Rapport pour le Service Développement Économique, 130 p.

Bruhat (T.), 1990. - Vingt technopoles. Un premier bilan, Paris, La Documentation française, coll. « Etudes DATAR », $214 \mathrm{p}$.

Damette (F.), Vire (E.), Godin (L.), Beckouche (P.), 1997. - La région du Nord-Pas-de-Calais : villes et système urbain, Lille, Agence de Développement et d’Urbanisme de Lille Métropole, 112 p.

Davezies (L.), 2004. - Évolution des villes nouvelles depuis 20 ans : Accueillir Produire Servir Desservir, Rapport financé par le PUCA dans le cadre du Programme Interministériel « Histoire et évaluation des villes nouvelles », $78 \mathrm{p}$.

Delbar (G.), 1977. - La ville nouvelle de Lille-Est, son insertion et son rôle dans les schémas d'aménagement de la région $d u$ Nord, Thèse de doctorat de $3^{\mathrm{e}}$ cycle de géographie physique et régionale, USTL/ Université de Lille 1, 224 p.

Dubrulle (A.), 1993. - Le réseau Technopole : une démarche technopolitaine pour la Métropole et la Région Nord-Pas-de-Calais. Une stratégie en émergence, USTL/Université de Lille 1, Atelier de Prospective et d'Évaluation Universitaire, Note ${ }^{\circ} 2,13$ p. (non publié).

EPALE, 1978. - Dossier « l'accueil des activités en ville nouvelle », Ville nouvelle actualités, Villeneuve-d'Ascq, $n^{\circ} 20,19 p$.

EstienNe (I.), 2004. - L'intervention du paysagiste dans la ville de 1960 à aujourd'hui, significations, pertinences et enjeux pour les architectes et les urbanistes, Mémoire de DEA de Géographie, USTL/Université de Lille 1, $120 \mathrm{p}$.

FACHe (J.), 2002. - Les territoires de la haute technologie, Rennes, Presses Universitaires de Rennes, coll. «Didact Géographie », 157 p.

Faucret (A.), Pacaux (N.), Walkiewicz (A.-M.), 1991. - L'analyse du tissu économique de Villeneuve-d'Ascq : étude de deux parcs d'activités, Mémoire de maîtrise de Géographie, USTL/Université de Lille 1, 130 p.

Florida (R.), 2002. - The Rise of the Creative Class, New York, Basic Books, 434 p.

Gregoris (M.), 1991. - Toulouse : de la métropole à la technopole, Thèse de doctorat en Géographie et aménagement, Université de Toulouse-Le-Mirail, 360 p.

InSEE Nord-Pas-de-Calais, 2002. - «Une approche de la haute technologie dans le Nord-Pas-de-Calais », Les pages de Profils, Lille, $\mathrm{n}^{\circ}$ 4, 4 p.

—, 2005 - «État des lieux des activités de Recherche et Développement en Nord-Pas-de-Calais », Les pages de Profils, Lille, $\mathrm{n}^{\circ}$ 7, 6 p. 
Liefooghe (C.), 1985. - Léanord et Vélec, deux entreprises innovatrices du Nord-Pas-de-Calais, Mémoire de maîtrise de Géographie, USTL/Université de Lille 1, 194 p.

—, 2002 - La diffusion spatiale des services aux entreprises. Application à l'ancien bassin minier du Nord-Pas-de-Calais, Thèse de doctorat en géographie, USTL/Université de Lille 1, tome 1 (398 p) et tome 2 (90 documents et 26 annexes).

MaY (N.), 2000. - «Localisation et mobilité des services d'assistance et de conseil aux entreprises : une lecture critique des analyses en terme de facteurs de localisation », Colloque Économie et socio-économie des services, perspectives internationales, USTL/Université de Lille 1, 22 et 23 juin, 28 p. (non publié)

Oream-Nord, 1969. - Aménagement d'une région urbaine : le Nord-Pas-de-Calais, Paris, La Documentation française, $422 \mathrm{p}$.

PARIS (D.), 1993. - La mutation inachevée. Mutation économique et changement spatial dans le Nord-Pas-deCalais, Paris, L’Harmattan, 365 p.

Paris (D.), STEvens (J.-F.), 2000. - Lille et sa région urbaine. La bifurcation métropolitaine, Paris, L'Harmat$\tan , 265 \mathrm{p}$.

Parreau (M.), 1996. - Histoire de la faculté des sciences de 1854 à 1970, ASA, USTL/Université de Lille 1.

STACHOWSKI (K.), 2002. - Entreprise-territoire : géographie de l'innovation technologique dans la région NordPas-de-Calais, Thèse de doctorat en géographie, USTL/Université de Lille 1, 338 p.

Stevens (J.-F.), 1995. - «Science et ville », Ville et économie, Agence de Développement et d'Urbanisme de la Métropole Lilloise, ${ }^{\circ}$ 6, p. 2-5.

Tremblay (R.), 2006. - «La qualité de vie des villes du savoir », Les chantiers de la géographie, Colloque du 50 anniversaire des Cahiers de géographie du Québec, Université Laval, 28-29 avril, 6 p. [www.cgq. ulaval.ca].

Cet article a été reçu le 21 mars 2006 et définitivement accepté le 28 septembre 2006.

\section{NOTA BENE}

Outre la bibliographie, cet article se fonde sur des entretiens réalisés entre septembre 2005 et janvier 2006. Nous remercions pour leur témoignage les acteurs de l'histoire de Villeneuve-d'Ascq: G. Caudron (maire de Villeneuve-d'Ascq de 1977 à 2001), J.-M. Stievenard (son adjoint puis successeur), J. Cortois (président de l'USTL de 1982 à 1987), C. Gachelin (UFR géographie-USTL), P. Legros (architecte à l'EPALE), P. Thieffry (directeur du développement économique à l'EPALE), $G$. Zeller (architecte à l'EPALE). Notre gratitude va également aux acteurs actuels du développement technopolitain: P. Motte (SEDAF), B. Teresinski et A. Tison (SAEM Haute-Borne), E. Potel (service de développement économique de Villeneuve-d'Ascq), M. Switek et D. Droma (service Relations Université-Entreprises, USTL), D. Leroy (CréInnov, USTL), M. Bechtet (CIEL), M. Cornu (MITI Incubateur), et la Régie des ruches du département du Nord. Nous avons également consulté avec profit les sites Internet suivants : www.digiport.org, www.eurasante.com, www.franceincubation.com, www.iemn.univ-lille 1.fr, www.miti.fr, www.parc-hauteborne.fr, www.rassemblementcitoyen.org, www. retis-innovation.fr, www.tlmciel.com, www.univ-lille1.fr/cre-innov, www.villenewvedascq.fr, www. villes-nouvelles.equipement.gouv.fr. 
TeXTeS HORS DOSSIER THÉMATIQUE 
